\title{
Linear sets from projection of Desarguesian spreads
}

\author{
Vito Napolitano, Olga Polverino, Giovanni Zini and Ferdinando Zullo
}

\begin{abstract}
Every linear set in a Galois space is the projection of a subgeometry, and most known characterizations of linear sets are given under this point of view. For instance, scattered linear sets of pseudoregulus type are obtained by considering a Desarguesian spread of a subgeometry and projecting from a vertex which is spanned by all but two director spaces. In this paper we introduce the concept of linear sets of $h$-pseudoregulus type, which turns out to be projected from the span of an arbitrary number of director spaces of a Desarguesian spread of a subgeometry. Among these linear sets, we characterize those which are $h$-scattered and solve the equivalence problem between them; a key role is played by an algebraic tool recently introduced in the literature and known as Moore exponent set. As a byproduct, we classify asymptotically $h$-scattered linear sets of $h$-pseudoregulus type.
\end{abstract}

MSC 2010: 51E20, 05B25, 51E22

Keywords: Projective space, Desarguesian spread, Linear set, MRDcode.

This research was partially supported by the Italian National Group for Algebraic and Geometric Structures and their Applications (GNSAGA - INdAM). The first and the last author were also supported by the project "VALERE: Vanvitelli pEr la RicErca" of the University of Campania "Luigi Vanvitelli".

\section{Introduction}

Let $q$ be a prime power, $\mathbb{F}_{q}$ be the finite field of order $q$, and $\mathbb{F}_{q^{n}}$ be the degree $n$ extension of $\mathbb{F}_{q} \cdot \mathbb{F}_{q^{-}}$-linear sets in a Galois space $\Lambda=\operatorname{PG}\left(r-1, q^{n}\right)$ are a 
natural generalization of subgeometries $\Sigma=\operatorname{PG}(s, q)$ of $\Lambda$, and have been intensively investigated in the last decades. The intrinsic theoretical interest towards linear sets has been boosted in the last years by their applications in a number of areas, where they are used to construct or characterize a wide variety of geometrical and combinatorial objects. These areas include, but are not limited to, linear codes, semifields, blocking sets, strongly regular graphs; see [13, 14, 28] and the references therein.

The aim of this paper is to investigate a family of linear sets of $\Lambda=$ PG $\left((h+1) t-1, q^{n}\right)$, with $h, t \geq 1$, obtained by considering a Desarguesian $(n-1)$-spread $\mathcal{D}$ of $\Sigma=\operatorname{PG}(n t-1, q)$ and projecting from the span of $n-h-1$ director spaces of $\mathcal{D}$. When $h=1$, maximum scattered $\mathbb{F}_{q}$-linear sets of pseudoregulus type have been characterized in these terms in [19, generalizing results of [15, 25. In particular, those linear sets are associated with exactly $\frac{q^{n t}-1}{q^{n}-1}$ pairwise disjoint lines (forming the pseudoregulus) and admit exactly two $(t-1)$-dimensional subspaces intersecting each of these lines in one point. When $h \geq 2$, linear sets of this type still have a similar structure, once we replace lines by $h$-dimensional subspaces. These subspaces form what we call a $h$-pseudoregulus, cf. Definition 3.1, they admit exactly $h+$ 1 distinct $(t-1)$-subspaces intersecting each element of the $h$-pseudoregulus in one point. Recently, in [6] a family of scattered linear sets has been detected by imposing further requests on their intersections with $h$-subspaces of $\Lambda$; they have been named $h$-scattered linear sets. Our main results are the following ones:

- we show examples of linear sets of $h$-pseudoregulus type, for any admissible value of $h, t, q$ and $n$ (Theorem 3.2);

- we provide an analytic description of all linear sets of this type (Theorem 3.6 );

- we establish a connection between $h$-scattered linear sets of $h$ pseudoregulus type and a recently introduced algebraic tool called Moore exponent set (Theorem 4.1);

- we classify asymptotically maximum $h$-scattered linear sets of $h$ pseudoregulus type (Theorem 4.5) .

The paper is organized as follows. Section 2 contains preliminary results and tools about Desarguesian spreads, linear sets, rank metric codes and Moore exponent sets. 
Section 3 is devoted to the description of linear sets of $h$-pseudoregulus type, as defined in Definition 3.1. In particular, we introduce in Theorem 3.2 a class of linear sets of $h$-pseudoregulus type for every $h \geq 1, t, n \geq 2$, and prime power $q$; we prove in Theorem 3.6 that, up to equivalence, all linear sets of $h$-pseudoregulus type are of this form.

In Section 4 we deal with those linear sets of $h$-pseudoregulus type which are maximum $h$-scattered. More precisely, in Theorem 4.1 we associate with each of them a Moore exponent set for $q$ and $n$; among the linear sets of $h$-pseudoregulus type, the maximum $h$-scattered ones are actually characterized by means of Moore exponent sets. Moreover, in Theorem 4.2 we show the uniqueness of the $h$-pseudoregulus for maximum $h$-scattered linear sets which are not subgeometries. As a consequence, we completely solve in Corollary 4.4 the equivalence issue for those linear sets. Finally, relying on the asymptotic results in [2] for Moore exponent sets, we give in Theorem 4.5 an asymptotic classification of maximum $h$-scattered linear sets of $h$-pseudoregulus type.

\section{Preliminary notions and results}

\subsection{Desarguesian spreads}

An $(n-1)$-spread of $\mathrm{PG}(n t-1, q)$ is a family $\mathcal{S}$ of mutually disjoint $(n-1)$ dimensional subspaces such that each point of $\mathrm{PG}(n t-1, q)$ belongs to an element of $\mathcal{S}$. Examples of spreads are the Desarguesian ones, which can be constructed as follows. Every point $P$ of $\mathrm{PG}\left(n-1, q^{n}\right)=\operatorname{PG}\left(V, \mathbb{F}_{q^{n}}\right)$ defines an $(n-1)$-dimensional subspace $X(P)$ of $\mathrm{PG}(n t-1, q)=\mathrm{PG}\left(V, \mathbb{F}_{q}\right)$ and $\mathcal{D}=\left\{X(P): P \in \mathrm{PG}\left(t-1, q^{n}\right)\right\}$ is a spread of $\mathrm{PG}(n t-1, q)$, called a Desarguesian spread (see [29, Section 25]). If $t>2$, the incidence structure $\Pi_{t-1}(\mathcal{D})$, whose points are the elements of $\mathcal{D}$ and whose lines are the $(2 n-1)$ dimensional subspaces of $\mathrm{PG}(n t-1, q)$ joining two distinct elements of $\mathcal{D}$, is isomorphic to $\mathrm{PG}\left(t-1, q^{n}\right)$. The structure $\Pi_{t-1}(\mathcal{D})$ is called the $\mathbb{F}_{q}$-linear representation of $\mathrm{PG}\left(t-1, q^{n}\right)$.

A way to obtain a Desarguesian $(n-1)$-spread of $\mathrm{PG}(n t-1, q)$ is the following. Let $\Sigma=\mathrm{PG}(n t-1, q)$ and $\Sigma^{*}=\mathrm{PG}\left(n t-1, q^{n}\right)$. Embed $\Sigma$ in $\Sigma^{*}$ in such a way that $\Sigma=\operatorname{Fix}(\Psi)$, where $\Psi \in \operatorname{P} \Gamma L\left(\Sigma^{*}\right)$ is a semilinear collineation of order $n$ of $\Sigma^{*}$ whose fixed points are the points of $\Sigma$.

Lemma 2.1. [18, Lemma 1] Let $S$ be a subspace of $\Sigma^{*}$. Then $\operatorname{dim} S=$ 
$\operatorname{dim}(S \cap \Sigma)$ if and only if $S=S^{\Psi}$.

Let $\Theta=\operatorname{PG}\left(t-1, q^{n}\right)$ be a subspace of $\Sigma^{*}$ such that $\Theta, \Theta^{\Psi}, \ldots, \Theta^{\Psi^{n-1}}$ span the whole space $\Sigma^{*}$. If $P$ is a point of $\Theta$, then, by Lemma 2.1. $X^{*}(P)=$ $\left\langle P, P^{\Psi}, \ldots, P^{\Psi^{n-1}}\right\rangle$ is a $(n-1)$-dimensional subspace of $\Sigma^{*}$ defining a $(n-1)$ subspace $X(P)=X^{*}(P) \cap \Sigma$ of $\Sigma$. As $P$ runs over the subspace $\Theta$ we get a set of $q^{n(t-1)}+q^{n(t-2)}+\cdots+q^{n}+1$ mutually disjoint $(n-1)$-dimensional subspaces of $\Sigma$. Such a set is denoted by $\mathcal{D}=\mathcal{D}(\Theta)$ and is a Desarguesian $(n-1)$-spread of $\Sigma$; see [29]. The $(t-1)$-dimensional spaces $\Theta, \Theta^{\Psi}, \ldots, \Theta^{\Psi^{n-1}}$ are uniquely defined by the Desarguesian spread $\mathcal{D}$, i.e. $\mathcal{D}(\Theta)=\mathcal{D}(X)$ if and only if $X=\Theta^{\Psi^{i}}$ for some $i \in\{0,1, \ldots, n-1\}$, and are called director spaces of $\mathcal{D}$ (cf. [19]). Proposition 2.2 will play a special role in what follows.

Proposition 2.2. [19, Remark 2.1] Let $\mathcal{S}$ be an $(n-1)$-spread of $\Sigma=$ $\mathrm{PG}(n t-1, q)$ embedded in $\Sigma^{*}=\mathrm{PG}\left(n t-1, q^{n}\right)$ in such a way that $\Sigma=$ Fix $(\Psi)$, where $\Psi$ is a semilinear collineation of $\Sigma^{*}$ of order $n$. If $H$ is a $(t-1)$-dimensional subspace of $\Sigma^{*}$ such that

- $\Sigma^{*}=\left\langle H, H^{\Psi}, \ldots, H^{\Psi^{n-1}}\right\rangle$,

- $X^{*} \cap H \neq \emptyset$ for each $(n-1)$-dimensional subspace $X^{*}$ of $\Sigma^{*}$ such that $X^{*} \cap \Sigma \in \mathcal{S}$,

then $\mathcal{D}(H)=\mathcal{S}$, i.e. $\mathcal{S}$ is a Desarguesian spread and $H$ is one of its director spaces.

\section{$2.2 \quad$ Linear sets}

Let $\Lambda=\operatorname{PG}\left(r-1, q^{n}\right)=\operatorname{PG}\left(V, \mathbb{F}_{q^{n}}\right), q=p^{h}, p$ prime. A set $L$ of points of $\Lambda$ is an $\mathbb{F}_{q}$-linear set of $\Lambda$ if it is defined by the non-zero vectors of an $\mathbb{F}_{q^{-}}$-subspace $U$ of $V$, i.e. $L=L_{U}=\left\{\langle u\rangle_{\mathbb{F}_{q^{n}}}: u \in U^{*}\right\}$. The linear set $L_{U}$ has rank $k$ if $\operatorname{dim}_{\mathbb{F}_{q}} U=k$. If $\Omega=\operatorname{PG}\left(W, \mathbb{F}_{q^{n}}\right)$ is a subspace of $\Lambda$ and $L_{U}$ is an $\mathbb{F}_{q}$-linear set of $\Lambda$, then $\Omega \cap L_{U}$ is an $\mathbb{F}_{q}$-linear set of $\Omega$. The subspace $\Omega$ of $\Lambda$ has weight $i$ in $L_{U}$ if $\operatorname{dim}_{\mathbb{F}_{q}}(U \cap W)=i$, and we write $\omega_{L_{U}}(\Omega)=i$. If $L_{U} \neq \emptyset$, we have $\left|L_{U}\right| \equiv 1(\bmod q)$ and

$$
\left|L_{U}\right| \leq q^{k-1}+q^{k-2}+\cdots+q+1 .
$$

An $\mathbb{F}_{q}$-linear set $L_{U}$ of $\Lambda$ of rank $k$ is scattered if all points of $L_{U}$ have weight one, or equivalently, if $L_{U}$ has maximum size $q^{k-1}+q^{k-2}+\cdots+q+1$. 
Theorem 2.3. [3, Theorem 4.2] A scattered $\mathbb{F}_{q}$-linear set of $\mathrm{PG}\left(r-1, q^{n}\right)$ has rank at most $r n / 2$.

A scattered $\mathbb{F}_{q}$-linear set $L$ of $\mathrm{PG}\left(r-1, q^{n}\right)$ of maximum rank $r n / 2$ is called a maximum scattered linear set. As a consequence of Theorem 2.3 one gets that maximum scattered $\mathbb{F}_{q}$-linear sets of $\mathrm{PG}\left(r-1, q^{n}\right)$ span the whole space $\mathrm{PG}\left(r-1, q^{n}\right)$.

If $\operatorname{dim}_{\mathbb{F}_{q}} U=\operatorname{dim}_{\mathbb{F}_{q^{n}}} V=r$ and $\langle U\rangle_{\mathbb{F}_{q^{n}}}=V$, then the linear set $L_{U}$ is a subgeometry of $\mathrm{PG}\left(V, \mathbb{F}_{q^{n}}\right)=\mathrm{PG}\left(r-1, q^{n}\right)$ isomorphic to $\mathrm{PG}(r-1, q)$. If $n=2$, then $L_{U}$ is a Baer subgeometry of $\mathrm{PG}\left(r-1, q^{2}\right)$.

In [20] the following characterization of $\mathbb{F}_{q}$-linear sets is given. Let $\Sigma=$ $\mathrm{PG}\left(S, \mathbb{F}_{q}\right)=\mathrm{PG}(k-1, q)$ be a subgeometry of $\Sigma^{*}=\mathrm{PG}\left(S^{*}, \mathbb{F}_{q^{n}}\right)=\mathrm{PG}(k-$ $\left.1, q^{n}\right), \Gamma=\operatorname{PG}\left(H, \mathbb{F}_{q^{n}}\right)$ be a $(k-r-1)$-dimensional subspace of $\Sigma^{*}$ disjoint from $\Sigma$, and $\Lambda=\operatorname{PG}\left(V, \mathbb{F}_{q^{n}}\right)=\operatorname{PG}\left(r-1, q^{n}\right)$ be an $(r-1)$-dimensional subspace of $\Sigma^{*}$ disjoint from $\Gamma$. Let $L=\left\{p_{\Gamma, \Lambda}(P)=\langle\Gamma, P\rangle \cap \Lambda: P \in \Sigma\right\}$ denote the projection of $\Sigma$ from $\Gamma$ to $\Lambda$. The subspaces $\Gamma$ and $\Lambda$ are the center and axis of the projection $p_{\Gamma, \Lambda}$, respectively.

Theorem 2.4. 20, Theorems 1 and 2] If $L$ is a projection of $\Sigma=\mathrm{PG}(k-1, q)$ from $\Gamma=\mathrm{PG}\left(k-r-1, q^{n}\right)$ to $\Lambda=\mathrm{PG}\left(r-1, q^{n}\right)$, then $L$ is an $\mathbb{F}_{q}$-linear set of $\Lambda$ of rank $k$ and $\langle L\rangle=\Lambda$. Furthermore, with the above notation, $L=L_{U}$, where

$$
U=(S+H) \cap V .
$$

Conversely, if $L$ is an $\mathbb{F}_{q}$-linear set of rank $k$ and $\langle L\rangle=\Lambda$, then either $L$ is a subgeometry of $\Lambda$ or, for each $(k-r-1)$-dimensional subspace $\Gamma$ of $\Sigma^{*}=\mathrm{PG}\left(k-1, q^{n}\right)$ disjoint from $\Lambda$, there exists a subgeometry $\Sigma$ of $\Sigma^{*}$ disjoint from $\Gamma$ such that $L=p_{\Gamma, \Lambda}(\Sigma)$.

Proposition 2.5 shows that the linear set $L=p_{\Gamma, \Lambda}(\Sigma)$ does not depend on the axis $\Lambda$ of the projection, i.e. if $\bar{\Lambda}$ is an $(r-1)$-dimensional subspace of $\Sigma^{*}$ disjoint from $\Gamma$, then $\bar{L}=p_{\Gamma, \bar{\Lambda}}(\Sigma)$ is projectively equivalent to $L=p_{\Gamma, \Lambda}(\Sigma)$.

Proposition 2.5. With the same notation as in Theorem 2.4, let $\bar{L}=L_{\bar{U}}$ be the $\mathbb{F}_{q}$-linear set obtained by projecting $\Sigma$ from $\Gamma$ to another subspace $\bar{\Lambda}=\operatorname{PG}\left(\bar{V}, \mathbb{F}_{q^{n}}\right)=\operatorname{PG}\left(r-1, q^{n}\right)$ of $\Sigma^{*}$. Then there exists an $\mathbb{F}_{q^{n}}$-linear isomorphism $\omega: V \rightarrow \bar{V}$ such that $\omega(U)=\bar{U}$ and hence $\varphi_{\omega}(L)=\bar{L}$, where $\varphi_{\omega}: \Lambda \rightarrow \bar{\Lambda}$ is the projectivity induced by $\omega$.

Proof. Since $\Lambda \cap \Gamma=\bar{\Lambda} \cap \Gamma=\emptyset$, we have $S^{*}=V \oplus H=\bar{V} \oplus H$ and hence the maps $\phi_{V}: v \in V \mapsto v+H \in S^{*} / H, \phi_{\bar{V}}: v \in \bar{V} \mapsto v+H \in S^{*} / H$ 


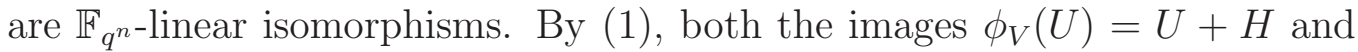
$\phi_{\bar{V}}(\bar{U})=\bar{U}+H$ are equal to $S+H$. Therefore, $\omega=\phi_{\bar{V}}^{-1} \circ \phi_{V}: V \rightarrow \bar{V}$ is an

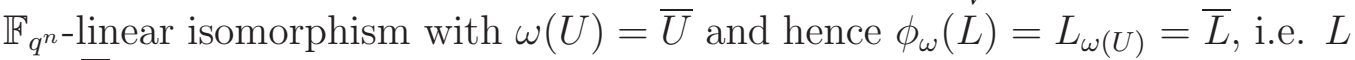
and $\bar{L}$ are projectively equivalent.

The concept of scattered linear sets was first generalized in [17, 32] to the concept of scattered linear sets with respect to the hyperplanes, and later in [6] as in Definition 2.6.

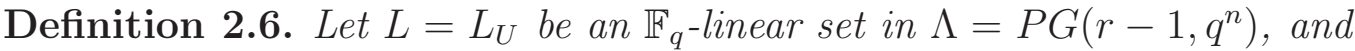
$h \leq r$ be a positive integer. The linear set $L$ is $h$-scattered if $\langle L\rangle=\Lambda$ and, for every $(h-1)$-dimensional subspace $\Omega$ of $\Lambda$, the weight $w_{L}(\Omega)$ is at most $h$. If $L$ is an $(r-1)$-scattered $\mathbb{F}_{q}$-linear set of $\Lambda$, then $L$ is also said to be scattered with respect to the hyperplanes.

Recall from [6, Proposition 2.1] that, if $L$ is a $h$-scattered linear set, then $L$ is also an $m$-scattered linear set whenever $1 \leq m \leq h$. Note also that 1-scattered linear sets of $\Lambda$ are exactly the scattered linear sets of $\Lambda$ which span $\Lambda$ over $\mathbb{F}_{q^{n}}$.

The following upper bound on the rank of a $h$-scattered linear set holds.

Theorem 2.7. [6, Theorem 2.3] If $L_{U}$ is a h-scattered linear set of rank $k$ in $\Lambda=\mathrm{PG}\left(r-1, q^{n}\right)$, then one of the following holds:

- $k=r$ and $L_{U}$ is a subgeometry $\mathrm{PG}(r-1, q)$ of $\Lambda$;

- $k \leq \frac{r n}{h+1}$.

A $h$-scattered linear set of maximum rank is said to be a maximum $h$ scattered linear set. Maximum $h$-scattered linear sets can be constructed by using the following result.

Theorem 2.8. [6, Theorem 2.5] Let $V=V_{1} \oplus \ldots \oplus V_{t}$, let $L_{U_{i}}$ be a $h_{i}$-scattered $\mathbb{F}_{q}$-linear set in $\mathrm{PG}\left(V_{i}, \mathbb{F}_{q^{n}}\right)$, with $i \in\{1, \ldots, t\}$ and let

$$
U=U_{1} \oplus \ldots \oplus U_{t}
$$

The $\mathbb{F}_{q}$-linear set $L_{U}$ is $h$-scattered in $\Lambda=\operatorname{PG}\left(V, \mathbb{F}_{q^{n}}\right)$, with $h=$ $\min \left\{h_{1}, \ldots, h_{t}\right\}$.

Furthermore, the projective equivalence for $h$-scattered linear sets coincides with the equivalence of the corresponding subspaces when $h \geq 2$. 
Theorem 2.9. [6, Theorem 4.5] Let $h \geq 2$. Two h-scattered $\mathbb{F}_{q}$-linear sets $L_{U}$ and $L_{W}$ of $\mathrm{PG}\left(r-1, q^{n}\right)$ are $\mathrm{P \Gamma L}\left(r, q^{n}\right)$-equivalent if and only if $U$ and $W$ are $\Gamma \mathrm{L}\left(r, q^{n}\right)$-equivalent.

\subsection{Rank metric codes}

Rank metric codes were introduced by Delsarte [9] in 1978 and they have been intensively investigated in recent years for their applications; we refer to 31] for a recent survey on this topic. The set of $m \times n$ matrices $\mathbb{F}_{q}^{m \times n}$ over $\mathbb{F}_{q}$ may be endowed with a metric, called rank metric, defined by

$$
d(A, B)=\operatorname{rk}(A-B)
$$

for any $A, B \in \mathbb{F}_{q}^{m \times n}$. A subset $\mathcal{C} \subseteq \mathbb{F}_{q}^{m \times n}$ equipped with the rank metric is called a rank metric code (or $R M$-code for short). The minimum distance of $\mathcal{C}$ is

$$
d=\min \{d(A, B): A, B \in \mathcal{C}, A \neq B\} .
$$

We are interested in $\mathrm{RM}$-codes which are $\mathbb{F}_{q}$-linear, i.e. $\mathbb{F}_{q}$-subspaces of $\mathbb{F}_{q}^{m \times n}$. In [9], Delsarte showed that the parameters of these codes must obey a Singleton-like bound, i.e.

$$
|\mathcal{C}| \leq q^{\max \{m, n\}(\min \{m, n\}-d+1)} .
$$

When the equality holds, we call $\mathcal{C}$ a maximum rank distance ( $M R D$ for short) code. Examples of $\mathbb{F}_{q}$-linear MRD-codes were first found by Delsarte in [9] and rediscovered by Gabidulin in [10]; although these codes have been originally found out by Delsarte, they are called Gabidulin codes since Gabidulin's publication contributed significantly to the development of rank metric codes. Two $\mathbb{F}_{q}$-linear RM-codes $\mathcal{C}$ and $\mathcal{C}^{\prime}$ are equivalent if and only if there exist $X \in \mathrm{GL}(m, q), Y \in \mathrm{GL}(n, q)$ and a field automorphism $\sigma$ of $\mathbb{F}_{q}$ such that

$$
\mathcal{C}^{\prime}=\left\{X C^{\sigma} Y: C \in \mathcal{C}\right\} .
$$

Let $\mathcal{C} \subseteq \mathbb{F}_{q}^{m \times n}$ be an RM-code, the adjoint code of $\mathcal{C}$ is

$$
\mathcal{C}^{\top}=\left\{C^{t}: C \in \mathcal{C}\right\} .
$$

Clearly, if $\mathcal{C}$ is an MRD-code then $\mathcal{C}^{\top}$ is MRD. 
Also, the left and right idealisers of $\mathcal{C}$ are defined as $L(\mathcal{C})=\{A \in$ $\mathrm{GL}(m, q): A \mathcal{C} \subseteq \mathcal{C}\}$ and $R(\mathcal{C})=\{B \in \mathrm{GL}(n, q): \mathcal{C} B \subseteq \mathcal{C}\}$. They are invariant under the equivalence of rank metric codes. Further invariants have been introduced in [11, 27]. In particular in [11, Section 4], the authors introduced the Gabidulin index of a rank metric code $\mathcal{C}$ as the maximum dimension of a subcode $\mathcal{G} \subseteq \mathcal{C}$ equivalent to a generalized Gabidulin code. The Gabidulin index has been calculated for the known families of MRD-codes with maximum left idealiser, see [11, Theorem 4.2].

Much of the focus on MRD-codes of $\mathbb{F}_{q}^{n \times n}$ to date has been on codes

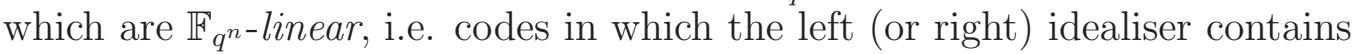
a field isomorphic to $\mathbb{F}_{q^{n}}$, since for these codes a fast decoding algorithm has been developed in [10]. Therefore, from a cryptographic point of view, it is very important to have many different examples of $\mathbb{F}_{q^{n}}$-linear MRD-codes. Unfortunately, very few examples are known, see [1, 4, 15, 8, 9, 10, 21, 24, 30, 33.

From now on, we identify $\mathbb{F}_{q}^{n \times n}$ with the algebra $\operatorname{End}_{\mathbb{F}_{q}}\left(\mathbb{F}_{q^{n}}\right)$. Since $\operatorname{End}_{\mathbb{F}_{q}}\left(\mathbb{F}_{q^{n}}\right)$ is isomorphic to the ring of $q$-polynomials over $\mathbb{F}_{q^{n}}$

$$
\mathcal{L}_{n, q}=\left\{\sum_{i=0}^{n-1} a_{i} x^{q^{i}}: a_{i} \in \mathbb{F}_{q^{n}}\right\},
$$

with addition and composition modulo $x^{q^{n}}-x$ as operations, we will consider an $\mathbb{F}_{q^{-}}$linear RM-code $\mathcal{C}$ as an $\mathbb{F}_{q^{-}}$-subspace of $\mathcal{L}_{n, q}$. In this setting, two $\mathbb{F}_{q^{-}}$ linear MRD-codes $\mathcal{C}_{1}$ and $\mathcal{C}_{2}$ are equivalent if and only if there exist two invertible $q$-polynomials $\varphi_{1}, \varphi_{2} \in \mathcal{L}_{n, q}$ and $\rho \in \operatorname{Aut}\left(\mathbb{F}_{q}\right)$ such that

$$
\varphi_{1} \circ f^{\rho} \circ \varphi_{2} \in \mathcal{C}_{2} \text { for all } f \in \mathcal{C}_{1}
$$

where $\circ$ stands for the composition of maps and $f^{\rho}(x)=\sum a_{i}^{\rho} x^{q^{i}}$ for $f(x)=$ $\sum a_{i} x^{q^{i}}$

The family of MRD-codes first found by Delsarte in [9], then by Gabidulin in [10] and generalized by Kshevetskiy and Gabidulin in [12], can be described as follows:

$$
\mathcal{G}_{k, s}=\left\langle x, x^{q^{s}}, \ldots, x^{q^{s(k-1)}}\right\rangle_{\mathbb{F}_{q^{n}}} \subseteq \mathcal{L}_{n, q}
$$

with $k \leq n-1$ and $\operatorname{gcd}(s, n)=1$.

Remark 2.10. Let $\operatorname{Tr}_{q^{n} / q}: \mathbb{F}_{q^{n}} \rightarrow \mathbb{F}_{q}$ be the trace map $x \mapsto x+x^{q}+\ldots+x^{q^{n-1}}$. The adjoint of a q-polynomial $f(x)=\sum_{i=0}^{n-1} a_{i} x^{q^{i}} \in \mathcal{L}_{n, q}$ with respect to the 
bilinear form $\langle x, y\rangle=\operatorname{Tr}_{q^{n} / q}(x y)$ is given by

$$
\hat{f}(x)=\sum_{i=0}^{n-1} a_{i}^{q^{n-i}} x^{q^{n-i}} .
$$

For a rank metric code $\mathcal{C}$ given by a set of linearized polynomials, its adjoint code may be seen as

$$
\mathcal{C}^{\top}=\{\hat{f}: f \in \mathcal{C}\},
$$

and the left and right idealisers of $\mathcal{C}$ can be written as:

$$
\begin{aligned}
& L(\mathcal{C})=\left\{\varphi \in \mathcal{L}_{n, q}: \varphi \circ f \in \mathcal{C} \text { for all } f \in \mathcal{C}\right\}, \\
& R(\mathcal{C})=\left\{\varphi \in \mathcal{L}_{n, q}: f \circ \varphi \in \mathcal{C} \text { for all } f \in \mathcal{C}\right\} .
\end{aligned}
$$

In the case of RM-codes generated by monomials, the equivalence problem is completely solved as follows.

Theorem 2.11. [5, Theorem 2.3] For $j=1,2$, let $I_{j}$ be a $k$-subset of $\{0, \ldots, n-1\}$ and

$$
\mathcal{C}_{j}=\left\langle x^{q^{i}}: i \in I_{j}\right\rangle_{\mathbb{F}_{q^{n}}} .
$$

Then $\mathcal{C}_{1}$ and $\mathcal{C}_{2}$ are equivalent if and only if

$$
I_{1}=I_{2}+s=\left\{i+s \quad(\bmod n): i \in I_{2}\right\}
$$

for some $s \in\{0, \ldots, n-1\}$.

Sheekey in [30] proved that some of these codes are connected to maximum scattered linear sets of the projective line; for further connections with linear sets see also [6, 7, 17, 32]. In particular, in [17, Section 2.7] and in [32] the connection between $\mathbb{F}_{q^{n}}$-linear MRD-codes and maximum $(r-1)$-scattered $\mathbb{F}_{q}$-linear sets in $\mathrm{PG}\left(r-1, q^{n}\right)$ has been pointed out, see also [6, Section 4.1].

Theorem 2.12. 32, Proposition 3.5] $\mathcal{C}$ is an $\mathbb{F}_{q}$-linear $M R D$-code of $\mathcal{L}_{n, q}$ with minimum distance $n-r+1$ and with left idealiser isomorphic to $\mathbb{F}_{q^{n}}$ if and only if, up to equivalence,

$$
\mathcal{C}=\left\langle f_{1}(x), \ldots, f_{r}(x)\right\rangle_{\mathbb{F}_{q^{n}}}
$$


for some $f_{1}, f_{2}, \ldots, f_{r} \in \mathcal{L}_{n, q}$ and the $\mathbb{F}_{q}$-subspace

$$
U_{\mathcal{C}}=\left\{\left(f_{1}(x), \ldots, f_{r}(x)\right): x \in \mathbb{F}_{q^{n}}\right\}
$$

defines a maximum $(r-1)$-scattered $\mathbb{F}_{q}$-linear set of $\mathrm{PG}\left(\mathbb{F}_{q^{n}}^{r}, \mathbb{F}_{q^{n}}\right)$. Furthermore, two $\mathbb{F}_{q}$-linear $M R D$-codes $\mathcal{C}$ and $\mathcal{C}^{\prime}$ of $\mathcal{L}_{n, q}$ with minimum distance $n-r+1$ of type (2) are equivalent if and only if $U_{\mathcal{C}}$ and $U_{\mathcal{C}^{\prime}}$ are $\Gamma \mathrm{L}\left(r, q^{n}\right)$ equivalent.

Remark 2.13. If

$$
\mathcal{C}=\left\langle f_{1}(x), \ldots, f_{r}(x)\right\rangle_{\mathbb{F}_{q^{n}}} \subseteq \mathcal{L}_{n, q}
$$

is an MRD-code, then

- $\operatorname{dim}_{\mathbb{F}_{q}} \operatorname{ker} f \leq r-1$ for each $f \in \mathcal{C} \backslash\{0\}$;

- for each $i \in\{0, \ldots, r-1\}$, there exists $g \in \mathcal{C}$ such that $\operatorname{dim}_{\mathbb{F}_{q}} \operatorname{ker} g=i$ (see e.g. [22, Lemma 2.1]).

\subsection{Moore exponent sets}

Let $I=\left\{i_{0}, i_{1}, \ldots i_{k-1}\right\} \subseteq \mathbb{Z}_{\geq 0}$. For every $A=\left(\alpha_{0}, \alpha_{1} \ldots, \alpha_{k-1}\right) \in \mathbb{F}_{q^{n}}^{k}$, denote

$$
M_{A, I}=\left(\begin{array}{cccc}
\alpha_{0}^{q^{i_{0}}} & \alpha_{0}^{q^{i_{1}}} & \cdots \cdots & \alpha_{0}^{q^{i_{k-1}}} \\
\alpha_{1}^{q^{i_{0}}} & \alpha_{1}^{q^{i_{1}}} & \cdots \cdots & \alpha_{1}^{q^{i_{k-1}}} \\
\vdots & \vdots & \ddots & \vdots \\
\alpha_{k-1}^{q^{i_{0}}} & \alpha_{k-1}^{q^{i_{1}}} & \cdots \cdots & \alpha_{k-1}^{q^{i_{k-1}}}
\end{array}\right) .
$$

The set $I$ is a Moore exponent set for $q$ and $n$ if

$\operatorname{det} M_{A, I}=0$ if and only if $\alpha_{0}, \alpha_{1} \ldots, \alpha_{k-1}$ are $\mathbb{F}_{q}$-linearly dependent.

The definition of Moore exponent set was introduced in [2].

Remark 2.14. If $k>n$, then two elements of $I$ are equal modulo $n$ and hence two columns of $M_{A, I}$ are the same. Thus, a Moore exponent set for $q$ and $n$ has size at most $n$. 
A first example of Moore exponent set is $I=\{0,1, \ldots, k-1\}$ for every prime power $q$ and every $n \geq k$; in such a case, $M_{A, I}$ is a square Moore matrix as originally introduced in [26]. For every $s \in \mathbb{N}$, a set $I$ of non-negative integers is a Moore exponent set for $q$ and $n$ if and only if $I+s=\{i+s$ $(\bmod n): i \in I\}$ is a Moore exponent set for $q$ and $n$. Thus, we may always assume that the smallest element in $I$ is 0 . Besides $I=\{0,1, \ldots, k-1\}$, the following are other known examples of Moore exponent sets.

- $I=\{0, d, \ldots,(k-1) d\}$ for any $q$ and $n$ satisfying $\operatorname{gcd}(d, n)=1$, that is, $I$ is given by the first elements of an arithmetic progression whose common difference is coprime with $n$; see [12].

- $I=\{0,1,3\}$ for $n=7$ and odd $q$; see [5].

- $I=\{0,1,3\}$ for $n=8$ and $q \equiv 1(\bmod 3)$; see [5].

- $I=\{0,2,3,4\}$, for $n=7$ and odd $q$; see $[5$.

- $I=\{0,2,3,4,5\}$, for $n=8$ and $q \equiv 1(\bmod 3) ;$ see $[5]$.

Theorem 2.15. [5, Theorem 2.5] Let $I=\left\{i_{0}, \ldots, i_{k-1}\right\}$ be a set of $k$ nonnegative integers. The following are equivalent:

- I is a Moore exponent set for $q$ and $n$;

- $\mathcal{C}=\left\langle x^{q^{i_{0}}}, x^{q^{i_{1}}}, \ldots, x^{q^{i_{k-1}}}\right\rangle_{\mathbb{F}_{q^{n}}} \subseteq \mathcal{L}_{n, q}$ is an MRD-code;

- $L_{U_{\mathcal{C}}}=\left\{\left\langle\left(x^{q^{i_{0}}}, x^{q^{i_{1}}}, \ldots, x^{q^{i_{k-1}}}\right)\right\rangle_{\mathbb{F}_{q^{n}}}: x \in \mathbb{F}_{q^{n}}^{*}\right\}$ is a scattered $\mathbb{F}_{q^{-} \text {-linear }}$ set with respect to the hyperplanes of $\mathrm{PG}\left(k-1, q^{n}\right)$.

Some easy consequences follow from Theorem 2.15. Let $h \geq 2, I=$ $\left\{0, i_{1}, \ldots, i_{h}\right\} \subseteq\{0, \ldots, n-1\}$; for $j=1, \ldots, h$, let $\sigma_{j}: \mathbb{F}_{q^{n}} \rightarrow \mathbb{F}_{q^{n}}$ be defined by $\sigma_{j}(x)=x^{q^{i_{j}}}$.

Proposition 2.16. If $I$ is a Moore exponent set for $q$ and $n$, then $\cap_{j=1}^{h} \operatorname{Fix}\left(\sigma_{j}\right)=\mathbb{F}_{q}$.

Proof. Let $\mathbb{F}_{q^{\ell}}=\cap_{j=1}^{h} \operatorname{Fix}\left(\sigma_{j}\right)$, so that $\ell$ divides $i_{j}$ for any $j$ and $\ell$ divides $n$. Let $\mathcal{C}=\left\langle x, x^{q^{i_{1}}}, \ldots, x^{q^{i_{h}}}\right\rangle_{\mathbb{F}_{q^{n}}}$ be the MRD-code associated with $I$. Then every $g \in \mathcal{C}$ is a $q^{\ell}$-polynomialm and hence $\operatorname{dim} \mathbb{F}_{q} \operatorname{ker} g$ is a multiple of $\ell$. As $\mathcal{C}$ is an MRD-code, by Remark 2.13, there exists $g \in \mathcal{C}$ with $\operatorname{dim}_{\mathbb{F}_{q}} \operatorname{ker} g=1$. This implies $\ell=1$, that is the claim. 
If $h=2$, Proposition 2.16 can be specialized as follows.

Proposition 2.17. Let $I=\left\{0, i_{1}, i_{2}\right\}$ be a Moore exponent set for $q$ and $n$. Then, either $\operatorname{Fix}\left(\sigma_{1}\right)=\mathbb{F}_{q}$ or $\operatorname{Fix}\left(\sigma_{2}\right)=\mathbb{F}_{q}$.

Proof. Assume to the contrary that $\operatorname{Fix}\left(\sigma_{1}\right)=\mathbb{F}_{q^{\ell}}$ and $\operatorname{Fix}\left(\sigma_{2}\right)=\mathbb{F}_{q^{m}}$, with $\ell, m>1$. Being $\left\{0, i_{1}, i_{2}\right\}$ a Moore exponent set, it follows that $\mathcal{C}=\left\langle x, x^{q^{i_{1}}}, x^{q^{i_{2}}}\right\rangle_{\mathbb{F}_{q^{n}}}$ is an MRD-code. Since $\operatorname{Fix}\left(\sigma_{j}\right)=\operatorname{ker}\left(x^{q^{i_{j}}}-x\right)$ for $j \in\{1,2\}$, one has that $\operatorname{dim}_{\mathbb{F}_{q}} \operatorname{ker}\left(x^{q^{i_{j}}}-x\right) \leq 2$ by Remark 2.13, hence $\ell=m=2$ and $n$ is even. It follows that $i_{1}$ and $i_{2}$ are even. Therefore, the elements in $\mathcal{C}$ are $q^{2}$-polynomials. Thus, we have a contradiction to Remark 2.13 .

Corollary 2.18. The Gabidulin index of an MRD-code $\mathcal{C}=\left\langle x, x^{q^{i_{1}}}, x^{q^{i_{2}}}\right\rangle_{\mathbb{F}_{q^{n}}}$ is greater than or equal to 2. In particular, if $\mathcal{C}$ is not equivalent to any generalized Gabidulin code, its Gabidulin index is two.

Proof. By Proposition 2.17, we have either $\operatorname{gcd}\left(i_{1}, n\right)=1 \operatorname{or} \operatorname{gcd}\left(i_{2}, n\right)=1$, i.e. either $\left\langle x, x^{q^{i_{1}}}\right\rangle_{\mathbb{F}_{q^{n}}}=\mathcal{G}_{2, i_{1}}$ or $\left\langle x, x^{q^{i_{2}}}\right\rangle_{\mathbb{F}_{q^{n}}}=\mathcal{G}_{2, i_{2}}$. The claim follows.

It was shown in [2] that, if $I$ is a Moore exponent set for $q$ and $n$ and $n$ is big enough with respect to the elements of $I$, then $I$ is given by the first elements of an arithmetic progression.

Theorem 2.19. [2, Theorems 1.1, 3.2 and 4.1] Let I be a Moore exponent set for $q$ and $n$, with $|I|>2$. Let $j$ be the largest element of $I$ and define $N$ to be either $4 j+2$ or $\frac{13}{3} j+2$ according to $|I|=3$ or $|I|>3$, respectively. If $q>5$ and $n>N$, then $I$ is given by the first elements of an arithmetic progression.

\section{Linear sets of $h$-pseudoregulus type}

The following definition generalizes the concept of linear set of pseudoregulus type.

Definition 3.1. Let $h \geq 1, t, n \geq 2$. An $\mathbb{F}_{q}$-linear set $L$ of rank $n t$ in $\Lambda=\mathrm{PG}\left((h+1) t-1, q^{n}\right)$ such that $\langle L\rangle=\Lambda$ is of $h$-pseudoregulus type if:

(a) there exist $s=\frac{q^{n t}-1}{q^{n}-1}$ pairwise disjoint $h$-subspaces $\pi_{1}, \ldots, \pi_{s}$ of $\Lambda$ such that $w_{L}\left(\pi_{j}\right)=n$ for any $j \in\{1, \ldots, s\}$; 
(b) there exist exactly $h+1$ distinct $(t-1)$-subspaces $T_{1}, \ldots, T_{h+1}$ of $\Lambda$ such that $\Lambda=\left\langle T_{1}, \ldots, T_{h+1}\right\rangle, T_{i} \cap \pi_{j} \neq \emptyset$ for any $i$ and $j$, and $L \cap K_{i_{0}}=\emptyset$ for any $i_{0} \in\{1, \ldots, h+1\}$, where $K_{i_{0}}=\left\langle\cup_{i \neq i_{0}} T_{i}\right\rangle$.

The set $\mathcal{P}=\left\{\pi_{1}, \ldots, \pi_{s}\right\}$ is called the $h$-pseudoregulus associated with $L$, and $T_{1}, \ldots, T_{h+1}$ are the transversal spaces of $\mathcal{P}$.

Note that from $\langle L\rangle=\Lambda$ follows $n \geq h+1$.

We start by showing examples of linear sets of $h$-pseudoregulus type; we will then prove that every linear set of $h$-pseudoregulus type is equivalent to one of such examples.

Theorem 3.2. Let $\Lambda=\operatorname{PG}\left(\mathbb{F}_{q^{n t}}^{h+1}, \mathbb{F}_{q^{n}}\right)=\operatorname{PG}\left((h+1) t-1, q^{n}\right)$, where $h, t, n$ are positive integers with $t, n \geq 2$ and $n \geq h+1$. For $j=2, \ldots, h+1$, let $f_{j}: \mathbb{F}_{q^{n t}} \rightarrow \mathbb{F}_{q^{n t}}$ be an invertible strictly $\mathbb{F}_{q^{n} \text {-semilinear map with companion }}$ automorphism $\sigma_{j} \in \operatorname{Gal}\left(\mathbb{F}_{q^{n}}: \mathbb{F}_{q}\right)$ such that $\sigma_{2}, \ldots, \sigma_{h+1}$ are pairwise distinct. Denote by $\underline{f}$ the $(h+1)$-tuple $\left(f_{1}=\mathrm{id}, f_{2}, \ldots, f_{h+1}\right)$. Let

$$
\begin{gathered}
U_{\underline{f}}=\left\{\left(x, f_{2}(x), \ldots, f_{h+1}(x)\right) \mid x \in \mathbb{F}_{q^{n t}}\right\}, \\
L_{\underline{f}}:=L_{U_{\underline{f}}}=\left\{\left\langle\left(x, f_{2}(x), \ldots, f_{h+1}(x)\right)\right\rangle_{\mathbb{F}_{q^{n}}} \mid x \in \mathbb{F}_{q^{n t}}^{*}\right\} \subset \Lambda .
\end{gathered}
$$

Then $L_{f}$ is an $\mathbb{F}_{q}$-linear set of $\Lambda$ of h-pseudoregulus type. Moreover, for any $x \in \mathbb{F}_{q^{n t}}^{*}$, define

$$
\begin{gathered}
W_{x}=\left\langle(x, 0, \ldots, 0),\left(0, f_{2}(x), \ldots, 0\right), \ldots,\left(0, \ldots, 0, f_{h+1}(x)\right)\right\rangle_{\mathbb{F}_{q^{n}}}, \\
\Pi_{x}=\operatorname{PG}\left(W_{x}, \mathbb{F}_{q^{n}}\right)=\operatorname{PG}\left(h, q^{n}\right) .
\end{gathered}
$$

For $i=1, \ldots, h+1$, let

$$
T_{i}=\operatorname{PG}\left(\left\langle\mathbf{e}_{i}\right\rangle_{\mathbb{F}_{q} n}, \mathbb{F}_{q^{n}}\right)=\operatorname{PG}\left(t-1, q^{n}\right) \subset \Lambda,
$$

where $\mathbf{e}_{i}$ is the unit $i$-th vector of the standard ordered basis of $\mathbb{F}_{q^{n t}}^{h+1}$. Then $\mathcal{P}=\left\{\Pi_{x} \mid x \in \mathbb{F}_{q^{n t}}^{*}\right\}$ is the h-pseudoregulus of $L_{\underline{f}}$ and $T_{1}, \ldots, T_{h+1}$ are the transversal spaces of $\mathcal{P}$.

First we show the following lemma.

Lemma 3.3. Let $x_{1}, \ldots, x_{s} \in \mathbb{F}_{q^{n t}}$. Then $x_{1}, \ldots, x_{s}$ are $\mathbb{F}_{q^{n}}$-independent if and only if $\operatorname{dim}\left\langle\Pi_{x_{1}}, \ldots, \Pi_{x_{s}}\right\rangle=(h+1) s-1$. Also, if $\Pi \in \mathcal{P}$ satisfies $\Pi \cap\left\langle\Pi_{x_{1}}, \ldots, \Pi_{x_{s}}\right\rangle \neq \emptyset$, then $\Pi \subseteq\left\langle\Pi_{x_{1}}, \ldots, \Pi_{x_{s}}\right\rangle$. 
Proof. Suppose $\operatorname{dim}\left\langle\Pi_{x_{1}}, \ldots, \Pi_{x_{s}}\right\rangle<(h+1) s-1$. Let $i \in\{1, \ldots, s\}$ such that there exists $P \in \Pi_{x_{i}} \cap\left\langle\Pi_{x_{j}}: j \neq i\right\rangle$. Write $P=$ $\left\langle\left(\lambda_{1} x_{i}, \ldots, \lambda_{\ell} f_{\ell}\left(x_{i}\right), \ldots, \lambda_{h+1} f_{h+1}\left(x_{i}\right)\right)\right\rangle_{\mathbb{F}_{q^{n}}}$. For some $\ell \in\{1, \ldots, h+1\}$, the $\ell$-th component of a vector defining $P$ is non-zero, i.e. $\lambda_{\ell} f_{\ell}\left(x_{i}\right) \neq 0$ and $\lambda_{\ell} f_{\ell}\left(x_{i}\right)=\sum_{j \neq i} \mu_{j} f_{\ell}\left(x_{j}\right)$ with $\mu_{j} \in \mathbb{F}_{q^{n}}$. This implies that $x_{1}, \ldots, x_{s}$ are $\mathbb{F}_{q^{n}}$-linearly dependent. Conversely, suppose that $x_{1}, \ldots, x_{s}$ are $\mathbb{F}_{q^{n-}}$ linearly dependent. Then there exists $i \in\{1, \ldots, s\}$ such that $x_{i}$ is an $\mathbb{F}_{q^{n}}$-linear combination of $\left\{x_{j}: j \neq i\right\}$. Thus, easy computations show that $\Pi_{x_{i}} \subseteq\left\langle\Pi_{x_{j}}: j \neq i\right\rangle$, and hence $\operatorname{dim}\left\langle\Pi_{x_{1}}, \ldots, \Pi_{x_{s}}\right\rangle<(h+1) s-1$. This also proves the last part of the claim.

Proof of Theorem 3.2. Let $x \in \mathbb{F}_{q^{n t}}^{*}$; we determine $\Pi_{x} \cap L_{\underline{f}}$. Let $P=$ $\left\langle\left(y, f_{2}(y), \ldots, f_{h+1}(y)\right)\right\rangle_{\mathbb{F}_{q^{n}}} \in L_{f} \cap \Pi_{x}$ for some $y \in \mathbb{F}_{q^{n t}}^{*}$. Then there exist $\lambda_{1}, \ldots, \lambda_{h+1} \in \mathbb{F}_{q^{n}}$ such that

$$
\left(\lambda_{1} x, \lambda_{2} f_{2}(x), \ldots, \lambda_{h+1} f_{h+1}(x)\right)=\left(y, f_{2}(y), \ldots, f_{h+1}(y)\right) .
$$

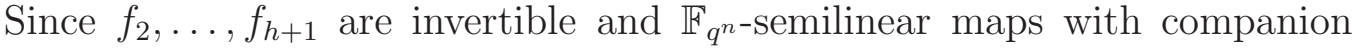
automorphisms $\sigma_{2}, \ldots, \sigma_{h+1}$ respectively, this is equivalent to

$$
\left\{\begin{array}{ccc}
y & = & \lambda_{1} x \\
\lambda_{2} & = & \lambda_{1}^{\sigma_{2}}, \\
& \vdots \\
\lambda_{h+1}= & \lambda_{1}^{\sigma_{h+1}} .
\end{array}\right.
$$

Thus, $L_{\underline{f}} \cap \Pi_{x}=\left\{\left\langle\left(\lambda_{1} x, \lambda_{1}^{\sigma_{2}} f_{2}(x), \ldots, \lambda_{1}^{\sigma_{h+1}} f_{h+1}(x)\right)\right\rangle_{\mathbb{F}_{q^{n}}} \mid \lambda_{1} \in \mathbb{F}_{q^{n}}^{*}\right\}$. Let

$$
W=\left\langle\mathbf{e}_{1}\right\rangle_{\mathbb{F}_{q^{n}}}+\ldots+\left\langle\mathbf{e}_{h+1}\right\rangle_{\mathbb{F}_{q^{n}}}=\mathbb{F}_{q^{n}}^{h+1} \subset \mathbb{F}_{q^{n t}}^{h+1} .
$$

Clearly, the map $F_{x}: W_{x} \rightarrow W$ defined by

$$
F_{x}\left(\lambda_{1} x, \ldots, \lambda_{h+1} f_{h+1}(x)\right)=\left(\lambda_{1}, \ldots, \lambda_{h+1}\right),
$$

is an invertible $\mathbb{F}_{q^{n}}$-linear map such that

$$
F_{x}\left(U_{\underline{f}} \cap W_{x}\right)=\left\{\left(\lambda, \lambda^{\sigma_{2}}, \ldots, \lambda^{\sigma_{h+1}}\right) \mid \lambda \in \mathbb{F}_{q^{n}}^{*}\right\} ;
$$

hence $L_{f} \cap \Pi_{x}$ and the linear set $\left\{\left\langle\left(\lambda, \lambda^{\sigma_{2}}, \ldots, \lambda^{\sigma_{h+1}}\right)\right\rangle_{\mathbb{F}_{q^{n}}} \mid \lambda \in \mathbb{F}_{q^{n}}^{*}\right\}$ are $\operatorname{PGL}\left(h+1, q^{n}\right)$-equivalent, and the weight of $\Pi_{x}$ with respect to $L_{f}$ is $n$. By Lemma 3.3, we get (a) of Definition 3.1. Also, since $\sigma_{2}, \ldots, \sigma_{h+1}$ are pairwise 
distinct, $\left\langle L \cap \Pi_{x}\right\rangle=\Pi_{x}$ for every $x \in \mathbb{F}_{q^{n t}}^{*}$ and hence $\langle L\rangle=\Lambda$. Clearly, $\Lambda=\left\langle T_{1}, \ldots, T_{h+1}\right\rangle$ and $T_{i} \cap \Pi_{x} \neq \emptyset$ for each $i=1, \ldots, h+1$ and $x \in \mathbb{F}_{q^{n t}}^{*}$.

Furthermore, $L_{f} \cap K_{i_{0}}=\emptyset$ for each $i_{0} \in\{1, \ldots, h+1\}$. Indeed, if $L_{f} \cap K_{i_{0}} \neq \emptyset$, then there exists $y \in \mathbb{F}_{q^{n t}}^{*}$ such that $f_{i_{0}}(y)=0$, a contradiction.

Now we prove that $T_{1}, \ldots, T_{h+1}$ are the unique transversal spaces of $\mathcal{P}$. Let $T$ be a transversal space; since by Definition $3.1 \mathcal{P}$ has exactly $\frac{q^{n t}-1}{q^{n}-1}$ pairwise disjoint elements and $|\mathcal{P}|=|T|$, we have that $T$ intersects every $\pi \in \mathcal{P}$ in exactly one point. As $\cap_{i=1}^{h+1} K_{i}=\emptyset$, there exists $\ell \in\{1, \ldots, h+$ $1\}$ such that $T \nsubseteq K_{\ell}$; we show that $T=T_{\ell}$. Let $P_{1}, \ldots, P_{t} \in T \backslash K_{\ell}$ be such that $T=\left\langle P_{1}, \ldots, P_{t}\right\rangle$. For any $i$, let $P_{i} \in \Pi_{x_{i}}$. For each $\Pi \in$ $\mathcal{P}$, we have $\Pi \cap T \neq \emptyset$; since $T \subseteq\left\langle\Pi_{x_{1}}, \ldots, \Pi_{x_{t}}\right\rangle$, Lemma 3.3 yields $\Pi \subseteq$ $\left\langle\Pi_{x_{1}}, \ldots, \Pi_{x_{t}}\right\rangle$. Thus, $\operatorname{dim}\left\langle\Pi_{x_{1}}, \ldots, \Pi_{x_{t}}\right\rangle=(h+1) t-1$ and hence, by Lemma

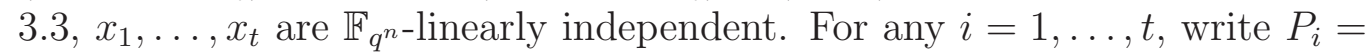
$\left\langle\left(\lambda_{x_{i}}^{(1)} x_{i}, \ldots, \lambda_{x_{i}}^{(\ell)} f_{\ell}\left(x_{i}\right), \ldots, \lambda_{x_{i}}^{(h+1)} f_{h+1}\left(x_{i}\right)\right)\right\rangle_{\mathbb{F}_{q^{n}}}$ with $\lambda_{x_{i}}^{(j)} \in \mathbb{F}_{q^{n}}$. Since $P_{i} \notin K_{\ell}$, we have $\lambda_{x_{i}}^{(\ell)} f_{\ell}\left(x_{i}\right) \neq 0$. As $x_{1}, \ldots, x_{t}$ and hence $f_{\ell}\left(x_{1}\right), \ldots, f_{\ell}\left(x_{t}\right)$ are $\mathbb{F}_{q^{n-}}$ linearly independent, we have $T \cap K_{\ell}=\emptyset$. Therefore, since $T \cap \Pi_{x} \neq \emptyset$ for every $x \in \mathbb{F}_{q^{n t}}^{*}, T=\operatorname{PG}\left(\tilde{W}, \mathbb{F}_{q^{n}}\right)$ with

$$
\tilde{W}=\left\{\left(\lambda_{x}^{(1)} x, \lambda_{x}^{(2)} f_{2}(x), \ldots, \lambda_{x}^{(h+1)} f_{h+1}(x)\right): x \in \mathbb{F}_{q^{n t}}\right\},
$$

where $\lambda_{x}^{(j)} \in \mathbb{F}_{q^{n}}$ and $\lambda_{x}^{(\ell)} \neq 0$ for every $x \in \mathbb{F}_{q^{n t}}^{*}$. Let $i \in\{1, \ldots, h+1\}$.

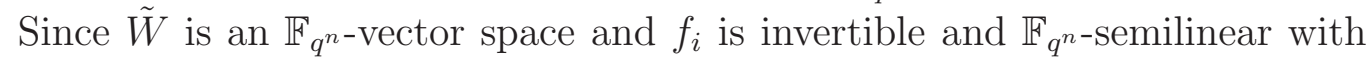
associated $\mathbb{F}_{q}$-automorphism $\sigma_{i}$, we have, for all $x, y \in \mathbb{F}_{q^{n t}}$,

$$
\lambda_{x}^{(i)} f_{i}(x)+\lambda_{y}^{(i)} f_{i}(y)=\lambda_{x+y}^{(i)} f_{i}(x+y),
$$

and hence

$$
\left(\left(\lambda_{x}^{(i)}\right)^{\sigma_{i}^{-1}}-\left(\lambda_{x+y}^{(i)}\right)^{\sigma_{i}^{-1}}\right) x=\left(\left(\lambda_{y}^{(i)}\right)^{\sigma_{i}^{-1}}-\left(\lambda_{x+y}^{(i)}\right)^{\sigma_{i}^{-1}}\right) y .
$$

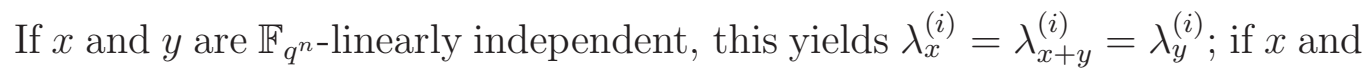
$y$ are $\mathbb{F}_{q^{n}}$-linearly dependent and $z \in \mathbb{F}_{q^{n t}} \backslash\langle x, y\rangle_{\mathbb{F}_{q^{n}}}$, then $\lambda_{x}^{(i)}=\lambda_{z}^{(i)}=\lambda_{y}^{(i)}$. Thus, $\lambda_{x}^{(i)}=\lambda^{(i)}$ is constant for all $x \in \mathbb{F}_{q^{n t}}$. Therefore,

$$
\tilde{W}=\left\{\left(\beta_{1} x, \ldots, f_{\ell}(x), \ldots, \beta_{h+1} g_{h+1}(x)\right): x \in \mathbb{F}_{q^{n t}}\right\}
$$

where $\beta_{i}=\lambda^{(i)} / \lambda^{(\ell)}$ for every $i \in\{1, \ldots, h+1\}$. Since $\tilde{W}$ is closed under

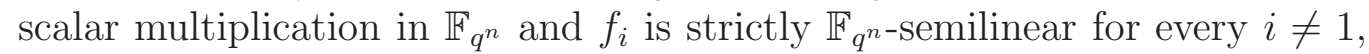
this implies that $\beta_{i}=0$ for $i \neq \ell$, i.e. $T=T_{\ell}$. 
Remark 3.4. It is readily seen from (6) that the slices $L_{f} \cap \Pi_{x}$ of the linear set $L_{f}$ lying on each space $\Pi_{x}$ are all PGL $\left((h+1) t, q^{n}\right)$-équivalent.

Remark 3.5. Let $V=V\left((h+1) t, q^{n}\right)=S_{1} \oplus \ldots \oplus S_{h+1}$ where $\operatorname{dim}_{\mathbb{F}_{q^{n}}} S_{i}=t$. For $i=2, \ldots, h+1$, let $g_{i}: S_{1} \rightarrow S_{i}$ be an invertible strictly $\mathbb{F}_{q^{n} \text {-semilinear }}$ map with companion automorphism $\sigma_{i} \in \operatorname{Gal}\left(\mathbb{F}_{q^{n}}: \mathbb{F}_{q}\right)$, such that $\sigma_{2}, \ldots, \sigma_{h+1}$ are pairwise distinct. Define

$$
\tilde{U}_{\underline{g}}=\left\{v+g_{2}(v)+\ldots+g_{h+1}(v) \mid v \in S_{1}\right\}
$$

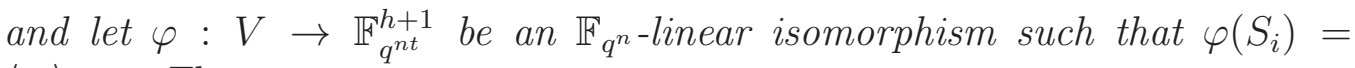
$\left\langle\mathbf{e}_{i}\right\rangle_{\mathbb{F}_{q n} \text {. Then }}$

$$
\varphi\left(\tilde{U}_{\underline{g}}\right)=U_{\underline{f}}
$$

where $f_{i}: \mathbb{F}_{q^{n t}} \rightarrow \mathbb{F}_{q^{n t}}$ is the $i$-th component of $\varphi \circ g_{i} \circ \varphi^{-1}(x, 0, \ldots, 0)$, and $f_{i}$ has companion automorphism $\sigma_{i}$.

In the following we will say that $\tilde{U}_{\underline{g}}$ and $U_{\underline{f}}$ are equivalent, because of (17).

\subsection{Analytic shape of a linear set of $h$-pseudoregulus type}

We now want to prove a converse of Theorem 3.2, that is, we show in the next result that every linear set of $h$-pseudoregulus type is equivalent to a linear set $L_{f}$. More precisely,

Theorem 3.6. Let $L$ be an $\mathbb{F}_{q}$-linear set of h-pseudoregulus type in $\Lambda=$ $\operatorname{PG}\left(V, \mathbb{F}_{q^{n}}\right)=\operatorname{PG}\left((h+1) t-1, q^{n}\right)$ and let $T_{i}=\operatorname{PG}\left(W_{i}, \mathbb{F}_{q^{n}}\right)$ be its transversal spaces for $i \in\{1, \ldots, h+1\}$. Then there exist invertible strictly $\mathbb{F}_{q^{n}-}$ semilinear maps $f_{i}: W_{1} \rightarrow W_{i}, i=2, \ldots, h+1$, with pairwise distinct companion $\mathbb{F}_{q}$-automorphisms, such that

$$
L=\left\{\left\langle w+f_{2}(w)+\ldots+f_{h+1}(w)\right\rangle_{\mathbb{F}_{q^{n}}}: w \in W_{1}^{*}\right\},
$$

hence $L$ is equivalent to a linear set of Form (41).

In order to prove Theorem 3.6, we need to look at the considered linear set as projection of a subgeometry.

Theorem 3.7. Let $\Sigma=\operatorname{PG}(n t-1, q)$ be a subgeometry of $\Sigma^{*}=$ $\mathrm{PG}\left(S^{*}, \mathbb{F}_{q^{n}}\right)=\operatorname{PG}\left(\right.$ tn $\left.-1, q^{n}\right), \Psi \in \mathrm{P} \Gamma \mathrm{L}\left(\Sigma^{*}\right)$ be a collineation of order $n$ such that $\operatorname{Fix}(\Psi)=\Sigma$. Let $i_{1}, \ldots, i_{h+1}$ be distinct elements of $\{0, \ldots, n-1\}$. Let $\mathcal{D}$ be a Desarguesian $(n-1)$-spread of $\Sigma$ with director subspace $\Theta$. Define 
- $\Gamma=\left\langle\Theta^{\Psi^{i}} \mid i \in\{0, \ldots, n-1\} \backslash\left\{i_{1}, \ldots, i_{h+1}\right\}\right\rangle$,

- $\Lambda=\left\langle\Theta^{\Psi^{i}} \mid i \in\left\{i_{1}, \ldots, i_{h+1}\right\}\right\rangle$.

Let $L$ be the projection of $\Sigma$ from $\Gamma$ to $\Lambda$. Then $L$ is an $\mathbb{F}_{q}$-linear set of h-pseudoregulus type in $\Lambda$ with transversal spaces $\Theta^{\Psi^{i_{1}}}, \ldots, \Theta^{\Psi^{i_{h+1}}}$ and $L$ is equivalent to $L_{\underline{f}}$ as in (41).

Proof. As $\mathcal{D}$ is a Desarguesian $(n-1)$-spread of $\Sigma$ with director space $\Theta$, we have that $\operatorname{dim} \Theta=t-1$, the director spaces of $\mathcal{D}$ are $\Theta, \Theta^{\Psi}, \ldots, \Theta^{\Psi^{n-1}}$, and $\Sigma^{*}=\left\langle\Theta, \Theta^{\Psi}, \ldots, \Theta^{\Psi^{n-1}}\right\rangle$; see Subsection 2.1. It follows that $\operatorname{dim} \Gamma=$ $(n-h-1) t-1$ and $\operatorname{dim} \Lambda=(h+1) t-1$. Since $\Sigma^{*}=\langle\Gamma, \Lambda\rangle$, this implies $\Gamma \cap \Lambda=\emptyset$. Also, $\Gamma \cap \Sigma=\emptyset$ because the $\Theta^{\Psi^{j}}$ 's are pairwise disjoint and cyclically permuted by $\Psi$. Therefore by Theorem 2.4 the projection $L=$ $p_{\Gamma, \Lambda}(\Sigma)$ is an $\mathbb{F}_{q}$-linear set of rank $n t$ in $\Lambda$ such that $\Lambda=\langle L\rangle$.

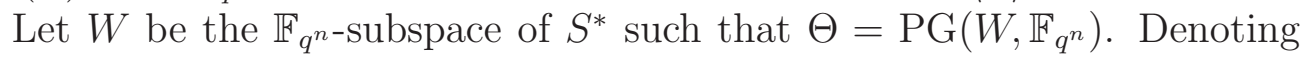
by $g \in \Gamma \mathrm{L}\left(S^{*}\right)$ the semilinear map associated with $\Psi$, we have

$$
\Sigma=\left\{\left\langle u+g(u)+\cdots+g^{n-1}(u)\right\rangle_{\mathbb{F}_{q^{n}}} \mid u \in W^{*}\right\} .
$$

In fact, the right-hand side of (8) is fixed by $\Psi$ elementwise and hence is contained in $\Sigma$; also, the dimension over $\mathbb{F}_{q}$ of both right- and left-hand side is equal to $n t-1$, because $\operatorname{dim}_{\mathbb{F}_{q^{n}}} W=t$. Thus,

$$
\begin{gathered}
L=p_{\Gamma, \Lambda}(\Sigma)=\left\{\left\langle g^{i_{1}}(u)+\cdots+g^{i_{h+1}}(u)\right\rangle_{\mathbb{F}_{q^{n}}} \mid u \in W^{*}\right\} \\
=\left\{\left\langle v+g^{i_{2}-i_{1}}(v)+\cdots+g^{i_{h+1}-i_{1}}(v)\right\rangle_{\mathbb{F}_{q^{n}}} \mid v \in g^{i_{1}}(W)^{*}\right\} .
\end{gathered}
$$

By Remark 3.5, $L$ is equivalent to $L_{f}$, where $f_{j}$ is the $j$-th component of $\varphi \circ g^{i_{j}-i_{1}} \circ \varphi^{-1}(x, 0 \ldots, 0)$ and $S_{j}=g^{i_{j}}(W)$. Denoting by $\sigma$ the companion automorphism of $g$, the automorphism associated with $f_{j}$ is $\sigma^{i_{j}-i_{1}}$, so that $f_{j}$ is not $\mathbb{F}_{q^{n} \text {-linear. }}$

Theorem 3.8. Let $L$ be an $\mathbb{F}_{q}$-linear set of rank $n t$ in $\Lambda=\operatorname{PG}\left((h+1) t-1, q^{n}\right)$ of $h$-pseudoregulus type with $h$-presudoregulus $\mathcal{P}$, having transversal spaces $T_{1}, \ldots, T_{h+1}$. Let $\Lambda$ be embedded in $\Sigma^{*}=\mathrm{PG}\left(t n-1, q^{n}\right)$. Let $\Sigma=\mathrm{PG}($ tn $1, q)$ be a subgeometry of $\Sigma^{*}, \Psi \in \operatorname{P\Gamma L}\left(\Sigma^{*}\right)$ be such that $\operatorname{Fix}(\Psi)=\Sigma$, and $\Gamma=\operatorname{PG}\left((n-h-1) t-1, q^{n}\right) \subseteq \Sigma^{*}$ be such that $L$ is the projection $p_{\Gamma, \Lambda}(\Sigma)$ from $\Gamma$ to $\Lambda$ of $\Sigma$. Then 
- the set $\mathcal{D}_{L}=\{\langle\Gamma, \pi\rangle \cap \Sigma \mid \pi \in \mathcal{P}\}$ is a Desarguesian $(n-1)$-spread of $\Sigma$;

- there exists a subset $\left\{\ell_{1}:=0, \ell_{2}, \ldots, \ell_{h+1}\right\}$ of $\{0,1, \ldots, n-1\}$, and a director space $\bar{\Theta}$ of $\mathcal{D}_{L}$, such that $\Gamma=\left\langle\bar{\Theta}^{\Psi^{j}} \mid j \notin\left\{0, \ell_{2}, \ldots, \ell_{h+1}\right\}\right\rangle$;

- $T_{i}=\left\langle\Gamma, \bar{\Theta}^{\Psi^{\ell_{i}}}\right\rangle \cap \Lambda$ for $i \in\{1, \ldots, h+1\}$.

Proof. For $\pi \in \mathcal{P}$, let $X_{\pi}=\langle\Gamma, \pi\rangle \cap \Sigma$. Since $L \cap \pi$ is an $\mathbb{F}_{q}$-linear set in $\pi$ of rank $n, X_{\pi}$ is an $(n-1)$-subspace of $\Sigma$. Also, by Definition 3.1(a), $\mathcal{D}_{L}$ is an $(n-1)$-spread of $\Sigma$. We show that $\mathcal{D}_{L}$ is Desarguesian.

Let $T_{1}, \ldots, T_{h+1}$ be the transversal spaces of $\mathcal{P}$. For any $i=1, \ldots, h+$ 1, define the subspaces $K_{i}=\left\langle\cup_{j \neq i} T_{j}\right\rangle$ and $\bar{K}_{i}=\left\langle\Gamma, K_{i}\right\rangle$ of $\Sigma^{*}$. Clearly, $\operatorname{dim} \bar{K}_{i}=(n-1) t-1$. Also, $\bar{K}_{i} \cap \Sigma=\emptyset$; in fact, if $Q \in \bar{K}_{i} \cap \Sigma$, then $\langle\Gamma, Q\rangle \cap \Lambda \in p_{\Gamma, \Lambda}(\Sigma)=L$ and $\langle\Gamma, Q\rangle \cap \Lambda \in \bar{K}_{i} \cap \Lambda=\left\langle\cup_{j \neq i} T_{j}\right\rangle$, a contradiction to $L \cap\left\langle\cup_{j \neq i} T_{j}\right\rangle=\emptyset((b)$ of Definiton 3.1). This implies that the $\Psi$-invariant subspace $\bar{K}_{i} \cap \bar{K}_{i}^{\Psi} \cap \cdots \cap \bar{K}_{i}^{\Psi^{n-1}}$ of $\bar{K}_{i}$ is empty.

Let $i=1$ and $\Theta=\bar{K}_{1} \cap \bar{K}_{1}^{\Psi} \cap \cdots \cap \bar{K}_{1}^{\Psi^{n-2}}$. An easy induction on $n$ proves that $\operatorname{dim} \Theta \geq t-1$. Together with $\Theta \cap \bar{K}_{1}^{\Psi^{n-1}}=\emptyset$, this yields $\operatorname{dim} \Theta=t-1$ and $\Sigma^{*}=\left\langle\Theta, \Theta^{\Psi}, \ldots, \Theta^{\Psi^{n-1}}\right\rangle$.

For any $\pi \in \mathcal{P}$, let $X_{\pi}^{*}=\left\langle X_{\pi}\right\rangle$ be the $(n-1)$-subspace of $\Sigma^{*}$ such that $X_{\pi}^{*} \cap \Sigma=X_{\pi}$; then $X_{\pi}^{*} \subseteq\langle\Gamma, \pi\rangle$. Since $X_{\pi}^{*} \cap \Sigma=\operatorname{PG}(n-1, q)$ and $\operatorname{dim} X_{\pi}^{*}=n-1$, Lemma 2.1 implies that $X_{\pi}^{*}$ is $\Psi$-invariant. Let $r=\pi \cap \bar{K}_{1}$. Since $\operatorname{dim} r=h-1$, we have that $\langle\Gamma, r\rangle$ is a hyperplane of $\langle\Gamma, \pi\rangle$. Also, $X_{\pi}^{*} \not \subset$ $\langle\Gamma, r\rangle$; otherwise, the non-empty subset $p_{\Gamma, \Lambda}\left(X_{\pi}\right)$ of $L$ would be contained in $\langle\Gamma, r\rangle \cap \Lambda=r \subset\left\langle\cup_{j \neq 1} T_{j}\right\rangle$, a contradiction by (b) of Definition 3.1. Thus, $H_{\pi}=X_{\pi}^{*} \cap\langle\Gamma, r\rangle$ is a hyperplane of $X_{\pi}^{*}$, i.e. $\operatorname{dim} H_{\pi}=n-2$. Since $H_{\pi} \subset \bar{K}_{1}$, we have $H_{\pi} \cap \Sigma=\emptyset$ and hence $H_{\pi} \cap H_{\pi}^{\Psi} \cap \cdots \cap H_{\pi}^{\Psi^{n-1}}=\emptyset$. So one gets that $H_{\pi} \cap H_{\pi}^{\Psi} \cap \cdots \cap H_{\pi}^{\Psi^{n-2}}$ is a point $R_{\pi}$ of $X_{\pi}^{*}$. Also, $R_{\pi} \in \Theta$ as $H_{\pi} \subset \bar{K}_{1}$. Therefore $R_{\pi} \in X_{\pi}^{*} \cap \Theta$, so that $X_{\pi}^{*} \cap \Theta$ is non-empty for any $\pi \in \mathcal{P}$. This implies that $\mathcal{D}_{L}$ is a Desarguesian spread of $\Sigma$ with director spaces $\Theta, \Theta^{\Psi}, \ldots, \Theta^{\Psi^{n-1}}$, see Proposition 2.2.

By direct computations, $\bar{K}_{1}=\left\langle\Theta^{\Psi^{j}} \mid j \neq 1\right\rangle$ and $\bar{K}_{1} \cap \Theta^{\Psi}=\emptyset$. Arguing in the same way we obtain for any $i=1, \ldots, h+1$ that $\bar{K}_{i} \cap \bar{K}_{i}^{\Psi} \cap \cdots \cap \bar{K}_{i}^{\Psi^{n-2}}$ is a director space for $\mathcal{D}_{L}$, hence there exists $i \in\{1, \ldots, h+1\}$ such that $\bar{K}_{i} \cap \bar{K}_{i}^{\Psi} \cap \cdots \cap \bar{K}_{i}^{\Psi^{n-2}}=\Theta^{\Psi^{\ell_{i}}}, \bar{K}_{i}=\left\langle\Theta^{\Psi^{j}} \mid j \neq \ell_{i}+1\right\rangle$, and $\bar{K}_{i} \cap \Theta^{\Psi^{\ell_{i}+1}}=\emptyset$; 
here, $\ell_{1}=0, \ell_{2}, \ldots, \ell_{h+1}$ are distinct integers in $\{0, \ldots, n-1\}$. Now we have

$$
\Gamma=\bigcap_{i=1}^{h+1} \bar{K}_{i}=\left\langle\Theta^{\Psi^{j}} \mid j \notin\left\{\ell_{1}+1, \ldots, \ell_{h+1}+1\right\}\right\rangle .
$$

Finally, for every $k \in\{1, \ldots, h+1\}$ we have $T_{k}=\bigcap_{i \neq k} K_{i}$ and

$$
\left\langle\Gamma, T_{k}\right\rangle \subseteq \bigcap_{i \neq k} \bar{K}_{i}=\bigcap_{i \neq k}\left\langle\Theta^{\Psi^{j}}: j \neq \ell_{i}+1\right\rangle=\left\langle\Gamma, \Theta^{\Psi^{\ell_{k}+1}}\right\rangle ;
$$

by comparing the dimensions, we get $\left\langle\Gamma, T_{k}\right\rangle=\left\langle\Gamma, \Theta^{\ell_{k}+1}\right\rangle$. It follows that $T_{k}=\left\langle\Gamma, \Theta^{\Psi^{\ell_{k}+1}}\right\rangle \cap \Lambda$. Writing $\bar{\Theta}=\Theta^{\Psi}$, the claim follows.

Proof of Theorem 3.6. By using the notations and the claims of Theorem 3.8, there exist $h+1$ distinct non-negative integers $\ell_{1}=0, \ell_{2}, \ldots, \ell_{h+1}$ and a director space $\bar{\Theta}=\mathrm{PG}\left(\bar{W}, \mathbb{F}_{q^{n}}\right)$ of $\mathcal{D}_{L}$ such that $T_{i}=\left\langle\Gamma, \bar{\Theta}^{\Psi^{\ell_{i}}}\right\rangle \cap \Lambda$ for every $i$. Define $\bar{\Lambda}=\left\langle\bar{\Theta}^{\Psi^{\ell_{j}}}: j \in\{1, \ldots, h+1\}\right\rangle=\operatorname{PG}\left(\bar{V}, \mathbb{F}_{q^{n}}\right)$. By Theorem 3.7, the $\mathbb{F}_{q}$-linear set $\bar{L}=p_{\Gamma, \bar{\Lambda}}(\Sigma)$ can be written as follows

$$
\bar{L}=\left\{\left\langle u+g^{\ell_{2}}(u)+\cdots+g^{\ell_{h+1}}(u)\right\rangle_{\mathbb{F}_{q^{n}}} \mid u \in \bar{W}^{*}\right\},
$$

where $g \in \Gamma \mathrm{L}\left(S^{*}\right)$ is the semilinear map associated with $\Psi$; see (9). By Proposition 2.5, there exists an $\mathbb{F}_{q^{n}}$-linear isomorphism $\omega: V \rightarrow \bar{V}$ such that $\omega(U)=\bar{U}$, where $L=L_{U}$ and $\bar{L}=L_{\bar{U}}$. The transversal spaces of the $h$ pseudoregulus $\overline{\mathcal{P}}$ of $\bar{L}$ are defined by $\bar{W}_{i}=g^{\ell_{i}}(\bar{W}), i=1, \ldots, h+1$. We have $\omega\left(W_{i}\right)=\bar{W}_{i}$, and hence the $\mathbb{F}_{q^{n}}$-semilinear invertible map $f_{i}=\omega^{-1} \circ g^{\ell_{i}} \circ \omega$ satisfies $f_{i}\left(W_{1}\right)=W_{i}$.

Therefore,

$$
L=\varphi_{\omega}^{-1}(\bar{L})=\left\{\left\langle w+f_{2}(w)+\ldots+f_{h+1}(w)\right\rangle_{\mathbb{F}_{q^{n}}}: w \in W_{1}^{*}\right\} .
$$

\section{Maximum $h$-scattered linear sets of $h$ - pseudoregulus type}

In this section we characterize maximum $h$-scattered linear sets of $h$ pseudoregulus type by means of Moore exponent sets of size $h+1$. For $h=1$, 
maximum 1-scattered linear set of 1-pseudoregulus type are exactly the classical maximum scattered linear sets of pseudoregulus type in $\mathrm{PG}\left(2 t-1, q^{n}\right)$, which have been characterized in [19, Theorem 3.5]. In this case, the only automorphism involved has the shape $x \in \mathbb{F}_{q^{n}} \mapsto x^{q^{s}} \in \mathbb{F}_{q^{n}}$ with $\operatorname{gcd}(s, n)=1$.

Let $h \geq 1$ and $t, n \geq 2$. For $j=2, \ldots, h+1$, let $f_{j}: \mathbb{F}_{q^{n t}} \rightarrow \mathbb{F}_{q^{n t}}$ be an

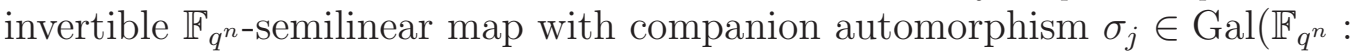
$\left.\mathbb{F}_{q}\right), \sigma_{j}: x \mapsto x^{q^{i_{j}}}$, where $i_{j} \in\{0, \ldots, n-1\}$; also, assume that the automorphisms $\sigma_{2}, \ldots, \sigma_{h+1}$ are pairwise distinct. Let $I=\left\{i_{1}=0, i_{2}, \ldots, i_{h+1}\right\}$. Define as in Section 3 the following objects: $\Lambda, \underline{f}, U_{\underline{f}}, L_{\underline{f}}, \Pi_{x}$ for any $x \in \mathbb{F}_{q^{n t}}^{*}$, $T_{1}, \ldots, T_{h+1}$.

We start this section by characterizing, among the linear sets of $h$ pseudoregulus type, the maximum $h$-scattered ones. Recall that, by Remark 3.5 and Theorem 3.6, every linear set of $h$-pseudoregulus type is equivalent to some $L_{\underline{f}}$ as in (41).

Theorem 4.1. Let $L$ be an $\mathbb{F}_{q}$-linear set of $h$-pseudoregulus type in $\mathrm{PG}((h+$ 1) $\left.t-1, q^{n}\right)$, where $L$ is equivalent to $L_{\underline{f}}$ as in (4). For every $j=2, \ldots, h+1$, let $i_{j} \in\{1, \ldots, n-1\}$ be such that $x \mapsto x^{q^{i_{j}}}$ is the companion automorphism of $f_{j}$; let $I=\left\{0, i_{2}, \ldots, i_{h+1}\right\}$. Then $L$ is maximum $h$-scattered if and only if $I$ is a Moore exponent set for $q$ and $n$.

Proof. Assume that $I$ is a Moore exponent set for $q$ and $n$. Let $x_{1}, \ldots, x_{t}$

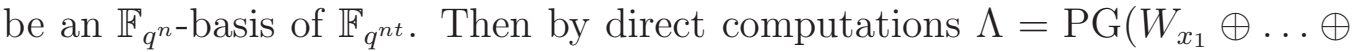
$\left.W_{x_{t}}, \mathbb{F}_{q^{n}}\right)$ and

$$
U_{\underline{f}}=\left(U_{\underline{f}} \cap W_{x_{1}}\right) \oplus \ldots \oplus\left(U_{\underline{f}} \cap W_{x_{t}}\right) .
$$

By ([6) $), U_{f} \cap W_{x_{i}}$ is $\operatorname{GL}\left(h+1, q^{n}\right)$-equivalent to $\left\{\left(\lambda, \lambda^{\sigma_{2}}, \ldots, \lambda^{\sigma_{h+1}}\right) \mid \lambda \in \mathbb{F}_{q^{n}}\right\}$. The latter defines a $h$-scattered $\mathbb{F}_{q^{-}}$-linear set in $\mathrm{PG}\left(h, q^{n}\right)$ because of Theorem 2.15; thus, $L_{f} \cap \Pi_{x_{i}}$ is a $h$-scattered $\mathbb{F}_{q}$-linear set in $\Pi_{x_{i}}$. From Theorem 2.8 and (10) follows that $L_{\underline{f}}$ is a $h$-scattered $\mathbb{F}_{q}$-linear set in $\Lambda$. As $L_{\underline{f}}$ has rank $n t$, Theorem 2.7 shows that $L_{f}$ is a maximum $h$-scattered linear set. The remaining parts of the claim follow from Theorem 3.2. Viceversa, assume that $L=L_{f}$ is a maximum $h$-scattered $\mathbb{F}_{q}$-linear set of $h$-pseudoregulus type in $\Lambda=\operatorname{PG}\left((h+1) t-1, q^{n}\right)$. Since $L$ is $h$-scattered, the linear set obtained as the intersection $L \cap \Pi$ of $L$ with an element $\Pi=\operatorname{PG}\left(h, q^{n}\right)$ of the $h$ pseudoregulus of $L$ is a $h$-scattered $\mathbb{F}_{q}$-linear set of rank $n$ of $\Pi$ equivalent to

$$
\left\{\left\langle\left(\lambda, \lambda^{\sigma_{2}}, \ldots, \lambda^{\sigma_{h+1}}\right)\right\rangle_{\mathbb{F}_{q^{n}}} \mid \lambda \in \mathbb{F}_{q^{n}}^{*}\right\} .
$$

By Theorem 2.15, this proves that $I$ is a Moore exponent set for $q$ and $n$. 
We show that every maximum $h$-scattered linear set in $\Lambda$ of $h$ pseudoregulus type, other than the subgeometries of $\Lambda$, has a unique $h$ pseudoregulus.

Theorem 4.2. Let $L$ be a maximum $h$-scattered $\mathbb{F}_{q}$-linear set in $\Lambda=\operatorname{PG}((h+$ $\left.1) t-1, q^{n}\right)$ of $h$-pseudoregulus type, with $h$-pseudoregulus $\mathcal{P}$. Then one of the following holds.

- $n=h+1, L$ is a subgeometry $\mathrm{PG}((h+1) t-1, q)$ of $\Lambda$; every Desarguesian $h$-spread of $L$ is a h-pseudoregulus associated with $L$, whose transversal spaces are the director spaces of the spread.

- $n>h+1$, then $w_{L}(\pi) \leq \frac{h n}{h+1}+1$ for any $h$-subspace $\pi$ of $\Lambda$ with $\pi \notin \mathcal{P}$; in particuar, $\mathcal{P}$ is the unique $h$-pseudoregulus associated with $L$.

Proof. Since $L$ is of $h$-pseudoregulus type we have $n \geq h+1$.

Suppose $n=h+1$. Then the rank of $L$ equals $\operatorname{dim} \Lambda+1$, and hence $L$ is a subgeometry $\mathrm{PG}((h+1) t-1, q)$ of $\Lambda=\mathrm{PG}\left((h+1) t-1, q^{h+1}\right)$. Let $\mathcal{D}$ be a Desarguesian $h$-spread of $L$. From the properties of Desarguesian spreads (see Subsection 2.1) follows immediately that $\mathcal{D}$ is a $h$-pseudoregulus for $L$, whose transversal spaces are its director spaces in $\Lambda$.

Suppose $n>h+1$. Let $\pi \notin \mathcal{P}$ be a $h$-subspace of $\Lambda$ with $\pi \cap L \neq \emptyset$, and $\Pi$ be an element of $\mathcal{P}$ such that $\Pi \cap \pi \neq \emptyset$. Define $k=\operatorname{dim}(\Pi \cap \pi) \in$ $\{0, \ldots, h-1\}$. Then the subspace $\langle\Pi, \pi\rangle$ of $\Lambda$ has dimension $2 h-k$. Together with Theorem 2.7 applied to the linear set $L \cap\langle\Pi, \pi\rangle$ in $\langle\Pi, \pi\rangle$, this implies

$$
w_{L}(\langle\Pi, \pi\rangle) \leq \frac{(2 h-k+1) n}{h+1} .
$$

On the other hand $\langle L \cap \Pi, L \cap \pi\rangle$ is a subspace of $L \cap\langle\Pi, \pi\rangle$, and since $L$ is $m$-scattered for each $1 \leq m \leq h$, we have

$$
w_{L}(\langle\Pi, \pi\rangle) \geq w_{L}(\Pi)-w_{L}(\pi)-w_{L}(\Pi \cap \pi) \geq n+w_{L}(\pi)-(k+1) .
$$

Therefore,

$$
w_{L}(\pi) \leq \frac{(h-k) n}{h+1}+k+1 .
$$

Since $n>h+1$, the right-hand side of (11) attains its maximum for $k=0$; in this case, $w_{L}(\pi) \leq \frac{h n}{h+1}+1$, so that $w_{L}(\pi)<n$. Thus, $\pi$ is not contained in any $h$-pseudoregulus for $L$, and the claim is proved. 
We are going to prove that the equivalence between two maximum $h$ scattered linear sets of $h$-pseudoregulus type only depends on the companion

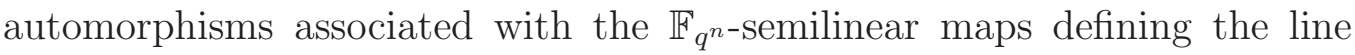
linear sets. For any $\mathbb{F}_{q}$-linear set $L_{\underline{f}}$ of $\Lambda$ as in (4), we denote by $A(\underline{f})=$ (id, $\left.\sigma_{2}, \ldots, \sigma_{h+1}\right)$ the $(h+1)$-ple of automorphisms associated with $\underline{f}$ and by $U_{A(\underline{f})}$ the $\mathbb{F}_{q^{-}}$vector space

$$
U_{A(\underline{f})}=\left\{\left(x, x^{\sigma_{2}}, \ldots, x^{\sigma_{h+1}}\right): x \in \mathbb{F}_{q^{n}}\right\} .
$$

Theorem 4.3. Two maximum $h$-scattered $\mathbb{F}_{q}$-linear sets of $h$-pseudoregulus type $L_{f}$ and $L_{g}$ in $\mathrm{PG}\left((h+1) t-1, q^{n}\right)$ are $\mathrm{P \Gamma L}\left((h+1) t, q^{n}\right)$-equivalent if and only if $U_{A(\underline{f})}$ and $U_{A(\underline{g})}$ are $\Gamma \mathrm{L}\left(h+1, q^{n}\right)$-equivalent.

Proof. By Theorem 2.9, $L_{f}$ and $L_{g}$ are $\operatorname{P\Gamma L}\left((h+1) t, q^{n}\right)$-equivalent if and only if $U_{f}$ and $U_{g}$ are $\Gamma \mathrm{L}\left((\bar{h}+1) t, \bar{q}^{n}\right)$-equivalent. Also, by Theorem 4.2, the pseudoreguli $\mathcal{P}_{\underline{f}}$ and $\mathcal{P}_{\underline{g}}$, associated respectively with $L_{\underline{f}}$ and $L_{\underline{g}}$, are uniquely determined.

If the linear sets $L_{f}$ and $L_{g}$ are $\operatorname{P\Gamma L}\left((h+1) t, q^{n}\right)$-equivalent, then by Theorem 2.9 there exists $F \in \bar{\Gamma} L\left((h+1) t, q^{n}\right)$ such that $F\left(U_{\underline{f}}\right)=U_{\underline{g}}$. Let $\Pi_{x_{i}}=\operatorname{PG}\left(W_{x_{i}}, \mathbb{F}_{q^{n}}\right)$, with $i \in\{1, \ldots, t\}$, be $t$ elements of $\mathcal{P}_{\underline{f}}$ such that

$$
U_{\underline{f}}=\left(W_{x_{1}} \cap U_{\underline{f}}\right) \oplus \ldots \oplus\left(W_{x_{t}} \cap U_{\underline{f}}\right) .
$$

Since $F\left(U_{f}\right)=U_{\underline{g}}$ and $\operatorname{dim}_{\mathbb{F}_{q}}\left(W_{x_{i}} \cap U_{\underline{f}}\right)=n$, we have that $\varphi_{F}\left(\Pi_{x_{i}}\right)=$ $\operatorname{PG}\left(F\left(W_{x_{i}}\right), \mathbb{F}_{q^{n}}\right) \in \mathcal{P}_{\underline{g}}$ and hence

$$
U_{\underline{g}}=\left(F\left(W_{x_{1}}\right) \cap U_{\underline{g}}\right) \oplus \ldots \oplus\left(F\left(W_{x_{t}}\right) \cap U_{\underline{g}}\right) .
$$

In particular, $F\left(W_{x_{1}} \cap U_{f}\right)=F\left(W_{x_{1}}\right) \cap U_{g}$. By (6) we have that $W_{x_{1}} \cap U_{f}$ is $\mathrm{GL}\left(h+1, q^{n}\right)$-equivalent to $U_{A(\underline{f})}$ and $F\left(\bar{W}_{x_{1}}\right) \cap U_{\underline{g}}$ is $\Gamma \mathrm{L}\left(h+1, q^{n}\right)$-equivalent to $U_{A(\underline{g})}$. Then the claim follows.

Conversely, assume that there exists $G \in \Gamma \mathrm{L}\left(\mathbb{F}_{q^{n t}}^{h+1}, \mathbb{F}_{q^{n}}\right)$ such that $G\left(U_{A(\underline{f})}\right)=U_{A(\underline{g})}$. As above, we can write

$$
U_{\underline{f}}=\left(W_{x_{1}} \cap U_{\underline{f}}\right) \oplus \ldots \oplus\left(W_{x_{t}} \cap U_{\underline{f}}\right),
$$

and

$$
U_{\underline{g}}=\left(W_{x_{1}}^{\prime} \cap U_{\underline{g}}\right) \oplus \ldots \oplus\left(W_{x_{t}}^{\prime} \cap U_{\underline{g}}\right),
$$


for some $\operatorname{PG}\left(W_{x_{i}}, \mathbb{F}_{q^{n}}\right) \in \mathcal{P}_{\underline{f}}$ and $\mathrm{PG}\left(W_{x_{i}}^{\prime}, \mathbb{F}_{q^{n}}\right) \in \mathcal{P}_{\underline{g}}$ with $x_{1}, \ldots, x_{t} \in \mathbb{F}_{q^{n t}}$ which are $\mathbb{F}_{q^{n}}$-linearly independent. By ([6) , for each $\bar{i} \in\{1, \ldots, t\}$ there exist two invertible $\mathbb{F}_{q^{n}}$-linear maps $F_{x_{i}}: W_{x_{i}} \rightarrow W$ and $H_{x_{i}}: W_{x_{i}}^{\prime} \rightarrow W$ such that

$$
F_{x_{i}}\left(W_{x_{i}} \cap U_{\underline{f}}\right)=U_{A(\underline{f})} \text { and } H_{x_{i}}\left(W_{x_{i}}^{\prime} \cap U_{\underline{g}}\right)=U_{A(\underline{g})},
$$

where $W=\mathbb{F}_{q^{n}}^{h+1}$. Define $\psi_{x_{i}}=H_{x_{i}}^{-1} \circ G \circ F_{x_{i}}: W_{x_{i}} \rightarrow W_{x_{i}}^{\prime}$. Since $V=$ $W_{x_{1}} \oplus \ldots \oplus W_{x_{t}}=W_{x_{1}}^{\prime} \oplus \ldots \oplus W_{x_{t}}^{\prime}$, if $\psi_{x_{i}}=H_{x_{i}}^{-1} \circ G \circ F_{x_{i}}: W_{x_{i}} \rightarrow W_{x_{i}}^{\prime}$, we may define the map

$$
\psi: V \rightarrow V, \quad \psi\left(\sum_{i=1}^{t} w_{i}\right)=\sum_{i=1}^{t} \psi_{x_{i}}\left(w_{i}\right), \quad \text { with } \quad w_{i} \in W_{x_{i}}
$$

Clearly, $\psi \in \Gamma L\left(V, \mathbb{F}_{q^{n}}\right)$ and

$$
\psi\left(U_{\underline{f}}\right)=U_{\underline{g}},
$$

so that $L_{\underline{f}}$ and $L_{\underline{g}}$ are $\operatorname{P\Gamma L}\left((h+1) t, q^{n}\right)$-equivalent.

Let id, $\sigma_{2}, \ldots, \sigma_{h+1}$ be the companion automorphisms associated with $\underline{f}$ and $I_{\underline{f}}=\left\{i_{1}=0, i_{2}, \ldots, i_{h+1}\right\}$ be such that $x^{\sigma_{j}}=x^{q^{i_{j}}}$ for each $j \in\{2, \ldots, h+$ $1\}$.

Corollary 4.4. Two maximum $h$-scattered $\mathbb{F}_{q}$-linear sets of $h$-pseudoregulus type $L_{f}$ and $L_{g}$ in $\mathrm{PG}\left((h+1) t-1, q^{n}\right)$ are $\mathrm{P} \Gamma \mathrm{L}\left((h+1) t, q^{n}\right)$-equivalent if and only if

$$
I_{\underline{f}}=I_{\underline{g}}+s=\left\{i+s \quad(\bmod n): i \in I_{\underline{g}}\right\},
$$

for some $s \in\{0, \ldots, n-1\}$.

Proof. By Theorem 4.3, $L_{f}$ and $L_{g}$ are $\operatorname{P\Gamma L}\left((h+1) t, q^{n}\right)$-equivalent if and only if $U_{A(\underline{f})}$ and $U_{A(\underline{g})}$ are $\bar{\Gamma} \mathrm{L}\left(h+\overline{1}, q^{n}\right)$-equivalent. By Theorem 2.15, $U_{A(\underline{f})}$ and $U_{A(\underline{g})}$ are $\Gamma \mathrm{L}\left(h+1, q^{n}\right)$-equivalent if and only if the MRD-codes $\mathcal{C}=$ $\left\langle x, x^{\sigma_{2}}, \ldots, x^{\sigma_{h+1}}\right\rangle_{\mathbb{F}_{q^{n}}}$ and $\mathcal{C}^{\prime}=\left\langle x, x^{\tau_{2}}, \ldots, x^{\tau_{h+1}}\right\rangle_{\mathbb{F}_{q^{n}}}$ are equivalent, where the $\sigma_{i}$ 's and the $\tau_{j}$ 's are the companion automorphisms of $\underline{f}$ and $\underline{g}$, respectively. From Theorem 2.11 the claim follows.

Finally, we point out that the asymptotic result of Theorem 2.19 about Moore exponent sets, together with Theorem 4.1 and Corollary 4.4, leads to an asymptotic structural classification of maximum $h$-scattered linear sets of pseudoregulus type. 
Theorem 4.5. Let $L$ be a maximum $h$-scattered $\mathbb{F}_{q}$-linear of rank $n t$ in $\Lambda=\mathrm{PG}\left((h+1) t-1, q^{n}\right)$ of $h$-pseudoregulus type, with $L$ equivalent to some $L_{f}$ as in (4). Let $I \subseteq\{0, \ldots, n-1\}$ be the Moore exponent set associated with $L_{\underline{f}}$, $j$ be the largest element of $I$, and

$$
N=\left\{\begin{array}{lll}
1 & \text { if } & h=1 \\
4 j+2 & \text { if } & h=2, \\
\frac{13}{3} j+2 & \text { if } & h>2 .
\end{array}\right.
$$

If $q>5$ and $n>N$, then there exists $s \in\{0, \ldots, n-1\}$ such that $\operatorname{gcd}(s, n)=$ 1 and $L$ is equivalent to

$$
\left\{\left\langle\left(x, x^{q^{s}}, x^{q^{2 s}} \ldots, x^{q^{h s}}\right)\right\rangle_{\mathbb{F}_{q^{n}}}: x \in \mathbb{F}_{q^{n t}}^{*}\right\} .
$$

Remark 4.6. For any $s, d \in\{1, \ldots, n-1\}$ coprime with $n$, recall that two generalized Gabidulin codes $\mathcal{G}_{k, s}$ and $\mathcal{G}_{k, d}$ are equivalent if and only if $s \equiv \pm d$ $(\bmod n) ;$ see [16, 23]. From Corollary 4.4 and Theorem 4.5 follows that, if $n>N$ and $q>5$, then there are exactly $\varphi(n) / 2$ orbits of $h$-scattered $\mathbb{F}_{q^{-}}$ linear sets of h-pseudoregulus type in $\Lambda$ under $\mathrm{P} \Gamma \mathrm{L}(\Lambda)$, where $\varphi$ is the Euler's totient function.

\section{References}

[1] D. Bartoli, C. Zanella And F. Zullo: A new family of maximum scattered linear sets in $\mathrm{PG}\left(1, q^{6}\right)$, arXiv:1910.02278.

[2] D. Bartoli And Y. Zhou: Asymptotics of Moore exponent sets, arXiv:1907.11100v1.

[3] A. Blokuis and M. Lavrauw: Scattered spaces with respect to a spread in PG(n,q), Geom. Ded. 81 (1-3), 231-243 (2000).

[4] B. Csajbók, G. Marino, O. Polverino and C. Zanella: A new family of MRD-codes, Linear Algebra Appl. 548 (2018), 203-220.

[5] B. Csajbók, G. Marino, O. Polverino and Y. Zhou: Maximum rank distance codes with maximum left and right idealisers, arxiv: 1807.08774 . 
[6] B. Csajbók, G. Marino, O. Polverino and F. Zullo: A special class of scattered subspaces, arxiv: 1906.10590.

[7] B. Csajbók, G. Marino, O. Polverino and F. Zullo: Maximum scattered linear sets and MRD-codes, J. Algebraic Combin. 46 (2017), 1-15.

[8] B. Csajbók, G. Marino and F. Zullo: New maximum scattered linear sets of the projective line, Finite Fields Appl. 54 (2018), 133-150.

[9] P. Delsarte: Bilinear forms over a finite field, with applications to coding theory, J. Combin. Theory Ser. A 25 (1978), 226-241.

[10] E. Gabidulin: Theory of codes with maximum rank distance, Problems of information transmission, 21(3) (1985), 3-16.

[11] L. Giuzzi And F. Zullo: Identifiers for MRD-codes, Linear Algebra Appl. 575 (2019), 66-86.

[12] A. Kshevetskiy and E. Gabidulin: The new construction of rank codes, International Symposium on Information Theory, 2005. ISIT 2005. Proceedings, pages 2105-2108, Sept. 2005.

[13] M. Lavrauw: Scattered spaces in Galois Geometry, Contemporary Developments in Finite Fields and Applications (2016), 195-216.

[14] M. Lavrauw and G. Van de Voorde: Field reduction and linear sets in finite geometry, In: Topics in Finite Fields, AMS Contemporary Math, vol. 623, pp. 271-293. American Mathematical Society, Providence (2015).

[15] M. Lavraum and G. Van de Voorde: Scattered linear sets and pseudoreguli, Electron. J. Combin. 20(1) (2013).

[16] D. Liebhold and G. Nebe: Automorphism groups of Gabidulin-like codes, Arch. Math. 107(4) (2016), 355-366.

[17] G. Lunardon: MRD-codes and linear sets, J. Combin. Theory Ser. A 149 (2017), 1-20.

[18] G. Lunardon: Normal spreads, Geom. Dedicata 75 (1999), 245-261. 
[19] G. Lunardon, G. Marino, O. Polverino and R. Trombetti: Maximum scattered linear sets of pseudoregulus type and the Segre variety $\mathcal{S}_{n, n}$, J. Algebr. Combin. 39, 807-831 (2014).

[20] G. Lunardon and O. Polverino: Translation ovoids of orthogonal polar spaces, Forum Math. 16, 663-669 (2004).

[21] G. Lunardon and O. Polverino: Blocking Sets and Derivable Partial Spreads, J. Algebraic Combin. 14 (2001), 49-56.

[22] G. Lunardon, R. Trombetti and Y. Zhou: On kernels and nuclei of rank metric codes, J. Algebraic Combin. 46 (2017), 313-340.

[23] G. Lunardon, R. Trombetti and Y. Zhou: Generalized Twisted Gabidulin Codes, J. Combin. Theory Ser. A 159 (2018), 79-106.

[24] G. Marino, M. Montanucci and F. Zullo: MRD-codes arising from the trinomial $x^{q}+x^{q^{3}}+c x^{q^{5}} \in \mathbb{F}_{q^{6}}[x]$, Linear Algebra Appl. 591 (2020), 99-114.

[25] G. Marino, O. Polverino and R. Trombetti: On $\mathbb{F}_{q}$-linear sets of PG( $\left(3, q^{3}\right)$ and semifields, J. Combin. Theory Ser. A 114 (2007), 769-788.

[26] E.H. Moore: A two-fold generalization of Fermats theorem, Bull. Amer. Math. Soc. 2(7) (1896), 189-199.

[27] A. Neri, S. Puchinger, A. Horlemann-Trautmann: Equivalence and Characterizations of Linear Rank-Metric Codes Based on Invariants, arXiv:1911.13059.

[28] O. Polverino: Linear sets in finite projective spaces, Discrete Math. 310(22) (2010), 3096-3107.

[29] B. Segre: Geometrie di Galois, fibrazioni proiettive e geometrie non desarguesiane, Ann. Mat. Pura Appl 64, 1-76 (1964).

[30] J. Sheekey: A new family of linear maximum rank distance codes, Adv. Math. Commun. 10(3) (2016), 475-488. 
[31] J. Sheekey: MRD codes: constructions and connections, Combinatorics and finite fields: Difference sets, polynomials, pseudorandomness and applications, Radon Series on Computational and Applied Mathematics, K.-U. Schmidt and A. Winterhof (eds.).

[32] J. Sheekey and G. Van de Voorde: Rank-metric codes, linear sets and their duality, Des. Codes Cryptogr. (2019), https://doi.org/10.1007/s10623-019-00703-z.

[33] C. Zanella AND F. Zullo: Vertex properties of maximum scattered linear sets of PG(1, $\left.q^{n}\right)$, Discrete Math. 343(5) (2020).

Vito Napolitano, Olga Polverino, Giovanni Zini and Ferdinando Zullo Dipartimento di Matematica e Fisica, Università degli Studi della Campania "Luigi Vanvitelli", Viale Lincoln 5, I-81100 Caserta, Italy \{vito.napolitano,olga.polverino,giovanni.zini,ferdinando.zullo\}@unicampania.it 